\title{
Linear oscillations of axisymmetric viscous liquid bridges
}

\author{
José $\Lambda$. Nicolás and José M. Vega
}

\begin{abstract}
Small amplitude free oscillations of axisymmetric capillary bridges are considered for varying values of the capillary Reynolds number $C^{-1}$ and the slenderness of the bridge $\Lambda$. A semi-analytical method is presented that provides cheap and accurate results for arbitrary values of $C^{1}$ and $\Lambda$ : several asymptotic limits (namely, $C \ll 1, C \gg 1, \Lambda \ll 1$ and $|\pi-\Lambda| \ll 1$ ) are considered in some detail, and the associatod approximate results are checked. $A$ fairly complete pieture of the (fairly complex) spoctrum of the linear problem is obtained for varying values of $C$ and $\Lambda$. ' $I$ 'wo kinds of normal modes, called capillary and hydrodynamic respectively, are almost always clearly identified, the former being associated with free surface deformation and the latter, only with the internal flow field; when $C$ is small the damping rate associated with both kind of modes is comparable, and the hydrodynamis ones explain the appearance of secondary (steady or slowly-varying) streaning hows.
\end{abstract}

Mathomatics Subjoct Classification (2000). 76Е09, 76L $205,76 \mathrm{D} 45,76 \mathrm{M} 25$.

Keywords. Surfaco-lension-driven instability, viscous damping, liquid bridges, linear ascillations.

\section{Introduction}

Licfuid bridges have been receiving a continued attention in the literature due to their intrinsic interest as basic configurations to understand some capillary and thermocapillary phenomena in microgravity, and to their applications in some natural phenomena and industrial processes. This paper is concerned with linear mechanical ascillations of liquid bridges for arbitrary values of the capillary Reynolds number $C^{1}$. Of course, the more interesting features associated with the spatio-temporal mechanical behavior of liquid bridges, such as breakage $\mid 1$, 2], hysteresis [3], chaotic behavior $[1,5]$ or streaming flows [3], [6]-[9], are absent when nonlinear terms are completely neglected. But the weakly-nonlinear description of that phenomena strongly relies on the precise qualitative and quantitative knowledge of lincar effects. Unfortunately, scveral basic issues on linear theory 
concerning both theory and comparison with experimentis remain uncovered, also for related fluid configurations (sce [10]-[16] and references given therein); we expect the basic ideas in this paper to also apply to these related problems.

l'revious work on linear oscillations of liquid bridges include the analyses of the strictly inviseid case $[17,18]$ and of the limits $C^{-1} \gg 1(|19|-|21|)$ and $C^{-1} \ll 1$ ([22]), and direct numerical computations for finite Reynolds numbers [23]. In particular, a boundary layer analysis was done by Borkar \& 'T'samopoulos [19] to calculate a first approximation of the damping rate that only accounted for viscous dissipation in the Stokes oscillatory boundary layers near the disks supporting the bridge; in order to doctor a strong discrepancy with experiments, a wetting boundary condition, with an adjustable parameter, was introduced for the free surface (even though the experiment suggested pinned ends). Such phenomenological condition was (implicitly) seen to be unnecessary by Tsamopoulos et al. [23], after a fully viscous, numerical computation with pinned-end boundary conditions that gave results that appeared to be in good agrement. with experiments [6]. The above-mentioned discrepancy in [19] was explained by IIiguera $e$ et al. [20] by just calculating a second approximation of the damping rate that also accounted for viscous dissipation in the bulk; in laxet we shall check below that this approximation gives reasonably good results for moderately large values of $C^{-1}$ (say, larger than 50), while the first approximation in $[19]$ is good only for unrealistically large values (say, $C^{-1} \geq 10^{6}$ ). The results in [23] were obtained by a finite element method that required to calculate the generalized eigenvalues of a large matrix $A$ and allowed fairly precise calculations in the range $3 \leq C^{-1} \leq 10^{3}$; the second limit was due to computer capability limitations (and could be socmingly improved today) but the first limit was not explained. Also, as we shall see in §3, Tsamopoulos el al. [23] ignored some overdamped eigenmodes (which will be called hydrodynamic bolow) exhibiting a damping rate that is comparable to that of the ones they considered (which will be called capillary).

In this paper wo shall consider the axisymmetric case, and use a semi-analytical method that consists of expanding the stream function as a series of products of trigonometric and Bessel functions. The main, well-known difficulty of the method is that the no-slip conditions at the disks cannot be satisfied by cach term in the expansion. The difliculty was solved by Joseph and co-workers [24]-[26] by using biorthogonal serites; the main idea involved (coming from the solution of a similar difliculty in elasticity problems $[27]$, was to expand the solution in terms of the eigenfunctions of an associated bilarmonic problem, which satisfies the no-slip conditions. After truncation to $N$ terms (truncation crrors are not easily estimated, see [24] and [26]) and elimination of spurious solutions [22], the eigenfrequencies $\Omega_{I}$ and damping rates $-\Omega_{R}$ are calculated by setting to zero the determinant of a complex $N \times N$ matrix $|22|-|23|$ whose coeflicients depend nonlinearly on $\Omega_{I}$ and $\Omega_{R}$. The main difficulties associated with this method come from the need of an initial guess, the significant role of truncation crrors [22] and the appearance of spurious wriggles even at, lower modes [23]. In this paper we shall 
require each term in the expansion to satisfy only one no-slip condition, and shall write down the more general solution not satisfying the other no-slip condition as the addition of two series; the other no-slip condition will be imposed to the whole serios expansion. In fact, the pinned-end boundary condition that must be imposed to the free surface involves an additional difliculty that also appeared and was already solved in the strictly inviscid case $[17,28]$. After truncation to $M+N$ terms (truncation errors will be easily estimated and convergenee will be casily improved, see [29]) the eigenfrequencies and damping rates will be calculated by solving a scalar, complex, truncated characterstic equation: in order to calculate the characteristic equation we shall only need to invert either a $M \times M$ or a $N \times N$ matrix. As we shall see, the method provides quite accurate and inexpensive results even for extreme values of the capillary Reynolds number, ranging from, say, $10^{-3}$ to $10^{4}$. The only problem is that we shall need an initial guess: we shall solve that difficulty by using approximate, asymptotic solutions for small or large values of the capillary Reynolds number (which will be also considered below), and applying a standard continualion lechnique $[30]$, which will also provide plots of the cigenfrequencies and damping rates in terms of (the capillary Reynolds number and) the slenderness of the bridge. Teet us point out here that the method in this paper is readily extended to obtain a semianalytical solution of more general linear problems, as will be explained below, at the end of $\$ 3.1$.

The paper is organized as follows. The governing equations will be formulated in $\$ 2$, and solved by the above-mentioned semi-analytical mothod in $\xi 3$, where the dependence of the eigenfrequency and damping rate on the slenderness and the capillary Reynolds number will be also analyzed. 'The asymptotic solutions in some limiting cases will be considered in $\$ 4$ and a comparison with approximale results from 1-D models [31] will be made in $\S 5$. Finally, several concluding remarks will be made in $\$ 6$.

\section{Formulation}

We consider a liquid bridge of length $L$, held by surface tension between two parallel, circular, coaxial disks of equal radii $R$. The density and viscosity of the surrounding gas are negligible as compared to the corresponding properties, $\rho$ and $\mu$, of the liquid (then the gas does not affect the dynamies of the liquid), and the latiter and the surface tension $\sigma$ are uniform and constant, and such that, the gravitational Bond number $B-\rho g R^{2} / \sigma(g-$ gravitational acceleration $)$ is appropriately small; then gravity will be neglected. Fintally, the free surface is anchored at the edges of the disks, the volume of the liquid equals that of the space in the cylinder bounded by the disks and we shall consider axisymmetric small-amplitude, free oscillations around the static cylindrical shape, and neglect, nonlinear corrections.

We shall use $R$ and the capillary time $\left(\rho R^{3} / \sigma\right)^{1 / 2}$ as characteristic length and 
time for nondimensionalization of the governing equations (continuity and momentum conscrvation) and boundary conditions (non-slipping at the disks, smoothnoss of the pressure and velocity fields at the axis of symmetry, kinematic compatibility and tangential and normal stress balanees at the free surface, and anchorage of the free surlace at the edges of the disks), which are writhen in terms of the shape of the free surface, $r-f(z, l)$, and the asual stream function, which for convencience will be written as rip, i.e,

$$
u--r^{1}{ }^{1}(r \varphi)_{z} \equiv-\varphi_{z}, \quad w-r^{1}(r \varphi)_{r} \equiv \varphi_{r}+r^{1}{ }_{\varphi}
$$

Ilere $u$ and $w$ are the (radial and axial) components of the velocity field in a cylindrical coordinate systom $(r, \theta, z)$, with its origin midway betwon the disks. If, in addition we linearize around the static state $(f-1, \varphi-0)$ and make a normal mode decomposition by sceking solutions of the form

$f(z, t)-1-\varepsilon F(z) \exp (\Omega t)+c . c+\ldots, \quad \varphi(r, z, t)-\varepsilon \phi(r, z) \exp (\Omega t)+c c+\ldots$,

where $s \rightarrow 0$ and a.c. stands for the complex conjugate, then $F$ and $\phi$ are seen to be given by the following linear eigenvalue problem

$$
\begin{aligned}
& (\mathcal{L}-\Omega / C) \mathcal{L} \phi=0 \quad \text { in } 0<r<1,-\Lambda<z<\Lambda, \\
& \phi-\phi_{z}-0 \text { a. } z- \pm \Lambda, \\
& \phi-\mathcal{L} \phi-0 \text { at } r-0, \\
& \phi_{z}\left|\Omega F^{\prime}=\phi_{r r}\right| \phi_{r}-\phi-\phi_{z z}=0 \text { at } r=1, \\
& F^{\prime \prime \prime} \mid F^{\prime \prime}=\Omega\left(\phi_{r} \mid \phi\right)-C\left(\phi_{r r r}\left|3 \phi_{r z z}\right| 3 \phi_{r r}\right) \text { at } r=1 \\
& F( \pm \Lambda)-\int_{\Lambda}^{\Lambda} F(z) \mathrm{d} z-0 .
\end{aligned}
$$

IIere the linear operator $\mathcal{L}$ is defined as

$$
\mathcal{L}=\left(\frac{\partial^{2}}{\partial r^{2}}\left|\frac{\partial^{2}}{\partial z^{2}}\right| r^{-1} \frac{\partial}{\partial r}-r^{-2}\right)
$$

and for convenience wo are imposing conservation of volume in (2.7). 'That linear problem depends only on two parameters, the capillary Reynolds number $C^{-1}$ ( $C$ is also called capillary number or Ohnesorge number) and the slernderness $\Lambda=L / 2 R$, which will be assumed to be smaller than $\pi$ to avoid the Rayleigh instability.

In addition to the normal modes given by (2.2)-(2.7), the system is readily seen to exhibit axisymmetric modes with non-xanishing azimuthal velocity v, which are readily calculated in closed-form (up to a constant factor) as

$u=w=p-1=0, \quad f-1=0, \quad v=\varepsilon J_{1}\left(s_{n} r\right) \sin \left[l_{m}(z \mid \Lambda)\right] \exp \left[-C\left(l_{m}^{2} \mid s_{n}^{2}\right) t\right]$, for $m, n-1,2, \ldots$, where $l_{m}-m \pi / 2 \Lambda$ and $s_{n}$ is a posilive rool of $s_{n} J_{1}^{\prime}\left(s_{n}\right)-$ $J_{1}\left(s_{n}\right), J_{1}$ being the second Bessel function. 


\section{Semi-analytical solution in the generic case}

For the sake of clarity, some involved expressions appearing in this section are given in the Appendix, at the end of the paper. The basic semianalytical solution to (2.2) (2.7) is obtained in $\$ 3.1$, where the scope of the method is also discussed. 'Then truncation errors are analyzed and convergenee is improved (in 33.2 ).

\subsection{Basic semianalytical solution}

The function $\psi-C \mathcal{L} \phi / \Omega$ satisfies (see (2.2) and (2.4))

$$
C \psi=\Omega 2 \psi / C ; \quad \psi=0 \text { at } r=0 \text {. }
$$

The general solution of that problem can be written as

$$
\begin{aligned}
w= & -\sum_{m=1}^{\infty} \frac{2 \alpha_{m} l_{m}^{2}}{l_{m}^{2}+\mu_{m}^{2}} \frac{I_{1}\left(l_{m}\right) I_{1}\left(\mu_{m} r\right)}{I_{0}\left(l_{m}\right) I_{1}\left(\mu_{m}\right)} \sin \left[l_{m}(z \mid \Lambda)\right] \\
& +\sum_{n=1}^{\infty}\left[\gamma_{n} \frac{\cosh \left(\nu_{n} z\right)}{\cosh \left(\nu_{n} \Lambda\right)}+\delta_{n} \frac{\sinh \left(\nu_{n} z\right)}{\sinh \left(\nu_{n} \Lambda\right)}\right] \frac{J_{1}\left(\lambda_{n} r\right)}{J_{0}\left(\lambda_{n}\right)}
\end{aligned}
$$

where $J_{0}, J_{1}, I_{0}$ and $I_{1}$ are respectively the first two Bessel functions and the first two modified Bessel functions: $\lambda_{1}, \lambda_{2}, \ldots$, are the positive roots of

$$
J_{1}\left(\lambda_{n}\right)-0
$$

and

$$
l_{m}-m \pi / 2 \Lambda, \quad \mu_{m}-\left(l_{m}^{2}+\Omega / C\right)^{1 / 2} ; \quad v_{n}-\left(\lambda_{n}^{2}+\Omega / C\right)^{1 / 2} .
$$

In order to obtain (3.2) we only noed to (a) consider (3.1) and the additional boundary conditions $\psi-g(z)$ at $r-1, \psi-f_{\perp}(r)$ at $z- \pm \Lambda$, and (b) write the unique solution of the resulting problem in terms of the Fourier coefficients of the cxpansions of $g$ and $f=$ in torms of the systems

$$
\left\{\sin \left[l_{m}(z \mid \Lambda)\right]\right\} \text { and }\left\{J_{1}\left(\lambda_{n} r\right) / J_{0}\left(\lambda_{n}\right)\right\}
$$

which are $\left\{-2 \alpha_{m} l_{m}^{2} I_{1}\left(l_{m}\right) /\left[\left(l_{m}^{2}+\mu_{m}^{2}\right) I_{0}\left(l_{m}\right)\right]\right\}$ and $\left\{\gamma_{n} \pm \delta_{n}\right\}$ respectively. Note that the systems (3.5) are complete and orthogonal with the obvious inner products.

The stream function $\phi$ satislies (see (2.3)-(2.4))

$$
\begin{gathered}
\mathcal{L} \phi=\Omega \psi / C \\
\phi_{r r}+\phi_{r}-\phi-\phi_{z z}-0 \text { a.t. } r-1, \quad \phi-0 \text { a.t. } r-0 \text { and } \mathrm{at}, z- \pm \Lambda
\end{gathered}
$$


That problem has a unique solution (if $\psi$ is given), which can be written as

$$
\begin{aligned}
\phi & =\sum_{m-1}^{\infty} \alpha_{m}\left[\frac{I_{1}\left(l_{m} r\right)}{I_{0}\left(l_{m}\right)}-\frac{2 l_{m}^{2}}{l_{m}^{2}+\mu_{m}^{2}} \frac{I_{1}\left(l_{m}\right) I_{1}\left(\mu_{m} r\right)}{I_{0}\left(l_{m}\right) I_{1}\left(\mu_{m}\right)}\right] \sin \left[l_{m}(z \mid \Lambda)\right] \\
& +\sum_{n=1}^{\infty} \gamma_{n}\left[\frac{\cosh \left(\nu_{n} z\right)}{\cosh \left(\nu_{n} \Lambda\right)}-\frac{\cosh \left(\lambda_{n} z\right)}{\cosh \left(\lambda_{n} \Lambda\right)}\right] \frac{J_{1}\left(\lambda_{n} r\right)}{J_{0}\left(\lambda_{n}\right)} \\
& +\sum_{n=1}^{\infty} \delta_{n}\left[\frac{\sinh \left(\nu_{n} z\right)}{\sinh \left(\nu_{n} \Lambda\right)}-\frac{\sinh \left(\lambda_{n} z\right)}{\sinh \left(\lambda_{n} \Lambda\right)}\right] \frac{J_{1}\left(\lambda_{n} r\right)}{J_{0}\left(\lambda_{n}\right)}
\end{aligned}
$$

At the moment wo have only imposed the first boundary condition (2.3), the boundary conditions (2.4) and the second boundary condition (2.5). The complex coefficients $\alpha_{m}, \gamma_{n}$ and $\delta_{n}$ and the function $F^{\prime}$ are calculated below by applying the second condition(s) (2.3) (which determines $\left\{\gamma_{n}\right\}$ and $\left\{\delta_{n}\right\}$ ), and the first condition (2.5) and conditions (2.6) (2.7) (which determine $\left\{\alpha_{m}\right\}$ and $F$ ).

The second boundary condition (2.3) is equivalont to impose that $\phi_{z}(r, \pm \Lambda)$ is orthogonal to $J_{1}\left(\lambda_{k} r\right)$ for all $k$, that is to say

$$
\int_{0}^{1} \phi_{z}(r, \pm \Lambda) J_{1}\left(\lambda_{k} r\right) r d r=0 \text { for all } k
$$

and when this condition is applied to (3.8) we obtain

$$
\begin{aligned}
& {\left[\operatorname{tanhh}\left(\lambda_{k} \Lambda\right)-\left(\nu_{k} / \lambda_{k}\right) \operatorname{lanh}\left(\nu_{k} \Lambda\right)\right] \gamma_{k}-\sum_{m o d d} A_{k m}^{1} \alpha_{m b},} \\
& \left\lfloor\left(\nu_{k} / \lambda_{k}\right) \operatorname{coth}\left(\nu_{k} \Lambda\right)-\operatorname{coth}\left(\lambda_{k} \Lambda\right) \mid \delta_{k}-\sum_{m \text { even }} A_{k m}^{1} \alpha_{m},\right.
\end{aligned}
$$

where $A_{k m}^{1}$ is given in the Appendix (eq. (A1)) and we have taken into account that $J_{1}^{\prime}\left(\lambda_{k}\right)-J_{0}\left(\lambda_{k}\right)$ (see (3.3)), eq. (3.4) and the well-known expressions for integrals of products of Bessel functions in, og., [33]). When taking into account (3.8), the frrst boundary condilion (2.5) yields

$$
F(z)--\sum_{m-1}^{\infty} A_{m}^{2} \alpha_{m} \cos \left[l_{m}(z+\Lambda)\right]
$$

where $A_{m}^{2}$ is given in the Appendix (eq. (A1)). On the other hand, if (3.8) is replaced in the right hand side of (2.6), the resulting equation is integrated and 
(3.4) is used, then we obtain

$$
\begin{aligned}
F(z)=K_{1} \sin z & \left|K_{2} \cos z\right| K_{3} \mid \sum_{n_{3}-1}^{\infty} A_{m}^{3}\left(l_{m}^{2}-1\right)^{-1} \alpha_{m} \cos \left[l_{m}(z \mid \Lambda)\right] \\
& +\sum_{n=1}^{\infty} A_{n}^{4}\left(\gamma_{n} \frac{\sinh \left(\lambda_{n} z\right)}{\cosh \left(\lambda_{n} \Lambda\right)}+\delta_{n} \frac{\cosh \left(\lambda_{n} z\right)}{\sinh \left(\lambda_{n} \Lambda\right)}\right) \\
& -\sum_{n=1}^{\infty} A_{n}^{5}\left(\gamma_{n} \frac{\sinh \left(\nu_{n} z\right)}{\cosh \left(\nu_{n} \Lambda\right)}+\delta_{n} \frac{\cosh \left(\nu_{n} z\right)}{\sinh \left(\nu_{n} \Lambda\right)}\right)
\end{aligned}
$$

where $A_{m}^{3}, A_{n}^{4}$ and $A_{n}^{5}$ are as given in the Appendix (eq. (A2)-(A3)) and the coefficients $K_{1}, K_{2}$ and $K_{3}$ are obtained upon application of conditions (2.7), as

$$
\begin{aligned}
& K_{1} \sin \Lambda-\sum_{m \text { odd }} A_{m}^{3}\left(l_{m}^{2}-1\right){ }^{1} \alpha_{m}-\sum_{n=1}^{\infty}\left[A_{n}^{4} \operatorname{lanhh}\left(\lambda_{n} \Lambda\right)-A_{n}^{5} \operatorname{lanh}\left(\nu_{n} \Lambda\right)\right]_{(3.14)}, \\
& K_{2}\left(\Lambda{ }^{1} \sin \Lambda-\cos \Lambda\right)= \\
& \quad \sum_{m \mathrm{even}} A_{m}^{3}\left(l_{m}^{2}-1\right)^{-1} \alpha_{m}+\sum_{n-1}^{\infty}\left[A_{n}^{1} \operatorname{coth}\left(\lambda_{n} \Lambda\right)-A_{n}^{5} \operatorname{coth}\left(\nu_{n} \Lambda\right)+A_{n !}^{6}\right] \delta_{n}, \\
& K_{3} \Lambda+K_{2} \sin \Lambda-\sum_{n=1}^{\infty} \Lambda A_{n}^{6} \delta_{n},
\end{aligned}
$$

where $A_{n 1}^{6}$ is given in the Appendix (eq. (A.3)). On the other hand, if the expressions (3.12) and (3.13) are identified (using the fouricr expansions of the various $z$-functions appearing in the right hand side of (3.13) in terms of the system $\left.\left\{\cos \left[l_{m}(z+\Lambda)\right]\right\}\right)$ then we obtain

$$
\begin{aligned}
& {\left[\Lambda_{m}^{3} \mid\left(l_{m}^{2}-1\right) \Lambda_{m}^{2}\right] \alpha_{m}=2 \Lambda^{1} K_{1} \cos \Lambda \mid \sum_{n=1}^{\infty} \Lambda_{m n}^{7} \gamma_{n} \text { if } m \text { is odd, and }} \\
& {\left[\Lambda_{m}^{3} \mid\left(l_{m}^{2}-1\right) \Lambda_{m}^{2}\right] \alpha_{m}=2 \Lambda^{-1} K_{2} \sin \Lambda-\sum_{n-1}^{\infty} \Lambda_{m n}^{7} \delta_{n} \text { if } m \geq 2 \text { is cven, }}
\end{aligned}
$$

where $A_{m n}^{7}$ is as given in the Appendix (eq. (A.4)).

Now, by collecting our results above we can see two uncoupled systems of infinitely many equations. Eqs. (3.10), (3.14) and (3.17) involve only the sequences $\left\{\alpha_{m}\right\}_{m-o d d}$ and $\left\{\gamma_{n}\right\}$, and the scalar $K_{1}$; by requiring that system to have a nontrivial solution we obtain a characteristic equation giving the eigenvalue $\Omega$. The associated eigenmodes are such that $\alpha_{m}-\delta_{n}-0$ for all $m-$ even and all $n$, and will be called odd modes. Similarly, eqs. (3.11), (3.15)-(3.16) and (3.18) 
involve only the sequences $\left\{\alpha_{m}\right\}_{m-c v e n},\left\{\delta_{n}\right\}$ and the scalar $K_{2}$ and provide a second characteristic equation for the cigenvalue $\Omega$; the associated cigenmodes are such $\alpha_{m}-\gamma_{n}-0$ for all $m$ - odd and all $n$, and will called even modes. Note that the shape of the interface $F$ is an odd function of the axial variable $z$ and the stream function $\phi$ is an even function (thus the axial velocity is even and the radial velocity and pressure are odd) for odd modes, and the opposite is true for even modes. 'The existence of both kinds of modes could have been anticipated if taking into account that the linear problem (2.2) (2.7) is invariant under the symmetries

$$
z \rightarrow-z, \quad F \rightarrow-F, \quad \phi \rightarrow \phi ; \quad \text { and } z \rightarrow-z, \quad F \rightarrow F ; \quad \phi \rightarrow-\phi .
$$

The two linear systerns posed by eqs (3.10), (3.14), (3.17) and eqs (3.11), (3.15)(3.16), (3.18) cannot be solved in closed form. Thus they will be solved approximately upon truncation and, in order to lessen the computational cost, convergence properties of the process will be improved below. But, lirst, two remarks about the ideas leading to the solution above are in order:

a. $\Lambda$ similar procedure could have been followed by first imposing the second condition (2.3), instead of the first one, when solving (3.6): then the general solution (3.2) of (3.1) should have been written in terms of the system $\left\{\cos \left[\ln _{3}(z \mid \Lambda)\right]\right\}$ and the subsequent, expressions (3.8)-(3.18) should have been modified accordingly.

b. 'The ideas above apply to more general linear problems, whenever the spatial geometry (e.g., a strip or a cylinder) is appropriate to separation of variables, but some of the boundary conditions cannot be satisfied by each term in the resulting sequence. Tr only those conditions that can be imposed term-by-term are considered, the general solulion of the resulting under-determined problem will depend on several sequences of undetermined coefficients, one for cach boundary condition that is not imposed. When those additional boundary conditions are imposed, a set of lincar systems of infinitely many cquations aro obtained to calculate (after truncation) the above-mentioned undetermined coeflicients. The two key steps in the process (which require some care) are the calculation of the general solution of the under-determined problem, and the imposition of the additional boundary conditions to that general solution.

\subsection{Truncation errors and convergence acceleration}

Equations (3.10) (3.11) and (3.17) (3.18) (with the expressions in eqs. (A.1)-(A.3) in the $\Lambda$ ppendix) readily imply that, as $m \rightarrow \infty$ and $n \rightarrow \infty$,

$\alpha_{m}=a / l_{m} \mid o\left(l_{m}{ }^{1}\right)$ if $m$ is odd, $\alpha_{m}=b / l_{m} \mid o\left(l_{m}{ }^{1}\right)$ if $m$ is even, $\gamma_{n} \rightarrow 0, \delta_{n} \rightarrow 0$, where

$$
a-4 C \Lambda \perp\left[K_{1} \cos \Lambda-\sum_{n=1}^{\infty} A_{n}^{8} \gamma_{n}\right], \quad b-4 C \Lambda 1\left[K_{2} \sin \Lambda+\sum_{n=1}^{\infty} A_{n}^{8} \delta_{n}\right],
$$


with $A_{t i}^{8}$ as given in eq. (A.4) in the Appendix. Note that these estimates imply that the series expansion (3.8) and its derivatives of any order are convergent if $0 \leq r<1$ and $-\Lambda<z<\Lambda$. Also, if the series in (3.10) (3.11), (3.14) (3.16) and (3.17)-(3.18) are truncated to $M$ and $N$ torms in the indexes $m$ and $n$, truncation errors are readily seen to be of the order of

$$
O\left(l_{M}^{-3}\right), \quad O\left(l_{M}^{-2}\right)+o\left(\lambda_{N}^{-2}\right) \text { and } o\left(l_{m} /\left(l_{m}^{2}+\lambda_{N}^{2}\right)\right)
$$

respectively, and the crucial step in order to improve convergence is to lessen truncation errors in the first series in the right hand sides of (3.14) (3.15). To this end we add and subtract the quantitics

$$
\sum_{m o d d} \Omega a / l_{m}^{3} \equiv(2 \Lambda / \pi)^{3} \Omega A a \quad \text { and } \quad \sum_{m \text { even }} \Omega b / l_{m}^{3} \equiv(2 \Lambda / \pi)^{3} \Omega B b
$$

to the right hand sides of (3.14) and (3.15) respectively, to rewrite these equations as

$$
\begin{aligned}
& K_{1} \sin \Lambda-\sum_{m \text { odd }} {\left[A_{m h}^{3}\left(l_{m}^{2}-1\right)^{-1} \alpha_{m}-\Omega a / l_{m}^{3}\right]+(2 \Lambda / \pi)^{3} \Omega A a } \\
&-\sum_{n=1}^{\infty}\left[A_{n}^{4} \tanh \left(\lambda_{n h} \Lambda\right)-A_{n}^{5} \operatorname{lithh}\left(\nu_{n} \Lambda\right)\right] \gamma_{m} \\
& K_{2}\left(\Lambda^{-1} \sin \Lambda-\cos \Lambda\right)-\sum_{m \operatorname{cven}}\left[A_{m}^{3}\left(l_{m}^{2}-1\right)^{-1} \alpha_{m}-\Omega b / l_{m}^{3}\right]+(2 \Lambda / \pi)^{3} \Omega B b \\
&+\sum_{n-1}^{\infty}\left[A_{n}^{4} \operatorname{coth}\left(\lambda_{n} \Lambda\right)-A_{n}^{5} \operatorname{coth}\left(\nu_{n} \Lambda\right)+A_{m}^{6}\right] \delta_{n},
\end{aligned}
$$

where

$$
A=\sum_{m \text { odd }} m^{-3} \simeq 1.051803 \text { and } B=\sum_{m \in \text { even }} m^{-3} \simeq .1502575 .
$$

Now the truncation error in the right hand sides of (3.21)-(3.22) (which will replace below (3.14) (3.15) is $O\left(l_{M}^{-3}\right)+o\left(\lambda_{N}^{-2}\right)$ (instead of the $\left[O\left(l_{M}^{-2}\right)+o\left(\lambda_{N}^{-2}\right)\right]$-error we had before (sce (3.20)).

Now, after truncation, from eq. (3.17) we readily obtain the vector $\left\{\alpha_{m}\right\}$ in terms of the vector $\left\{\gamma_{n}\right\}$ and the scalar $K_{1}$. Substitution of this expression into (3.10) leads to a linear system of $N$ equations that can be solved to oblain $\left\{\gamma_{n}\right\}$ in terms of $K_{1}$ (that problem has been checked to be non singular for all tested values of the parameters). Then we only need to substitute the resulting expressions for $\left\{\alpha_{m u}\right\},\left\{\gamma_{n}\right\}$ and (3.19) into (3.21) to obtain a truncated characteristic equation, for odd modes of the type

$$
H_{M, N}^{\mathrm{odd}}(\Omega, \Lambda, O)-0 .
$$


Note that in order to obtain (3.17) we only need to invert a $N \times N$ matrix. Altornatively, we could begin, after truncation, by replacing (3.10) into (3.17), solve the resulting system of $(M+1) / 2$ equations and substitute its solution and (3.10) and (3.19) into (3.21); then we would need to invert a $[(M \mid 1) / 2]$-order matrix. A similar procedure applied to eqs. (3.11), (3.18), (3.19) and (3.22) leads to a truncaled characteristic equation for even modes,

$$
H_{M ; N}^{\mathrm{even}}(\Omega, \Lambda, C)=0
$$

Before proceeding further we point out that the characteristic equations (3.24)(3.25) exhibit spurious solulions that must be eliminated and are seen to occur when $\nu_{n}^{2}+1-0$, for some positive integer $n$; according to the definitions (3.4), these spurious solutions are $\Omega--C\left(1+\lambda_{n}^{2}\right)$.

In order to calculate $\Omega$ from (3.24) and (3.25) we do not use a Newton's method because to calculate the derivatives of $H_{M, N}$ is a tedious task. Instead, we employ a false position (or regula falsi) method [34] in the complex plane, which is fairly fast (two sleps of the method are roughly equivalent to one step of Newton's), bul. we need an initial estimate; the second estimate required to initiate the iterative process may bo taken as any value of $\Omega$ that is close to the estimate. For comparison we give our approximalion of the first eigenvalue in Table 1, for two pairs of values of the truncation parameters $M$ and $N$, along with the approximations by 'I'samopoulos et al. [23] (which required to calculate the generalized cigenvalues of a $899 \times 899$ matrix), by Borkar \& Tsamopoulos [19], and IIiguera et al. [20] and by Nicolás [22] (which apply to large and small values of the capillary Reynolds number $C^{-1}$ respectively). Note that there is a diflerence on the definition of the slenderness in this paper and in [23]; we have selected the value $\Lambda-\pi / 4$ that corresponds to most numerical results in [23]. In order to check relativo crrors in both frequency and damping rate, the results for $M-N-8$ and 32 are writton with precisely the number of digits that are exact. Lote that convergenee is quite fast as $M$ and $N$ increase, and that the results in [23] also provide fairly good results in the interval $2.4 \overline{5} \leq C^{-1} \leq 10^{3}$. Finally, the approximations in [20] and [22] provide reasonably good results (for $C^{1} \geq 50$ and $C^{1} \leq 1$ respectively), but the first approximation in [19] highly underestimates damping, even for $C^{1}=10^{4}$. These results illustrate that we can calculate the eigenvalues with extreme precision, at a reasonable computational cost. This lact is important, to ensure that some unexpected behavior of the eigenvalues in our results below is not due to lack of precision. In fact, all results below are exact up to the precision. of the plots.

\subsection{Continuation of solutions and basic results}

If the ralse position method is combined with a pseudo-arclength, continuation. method (see, e.g., [30]), then the initial estimate for each iteration is obtained in a. natural way (by means of, e.g., second order extrapolation based on the last, 


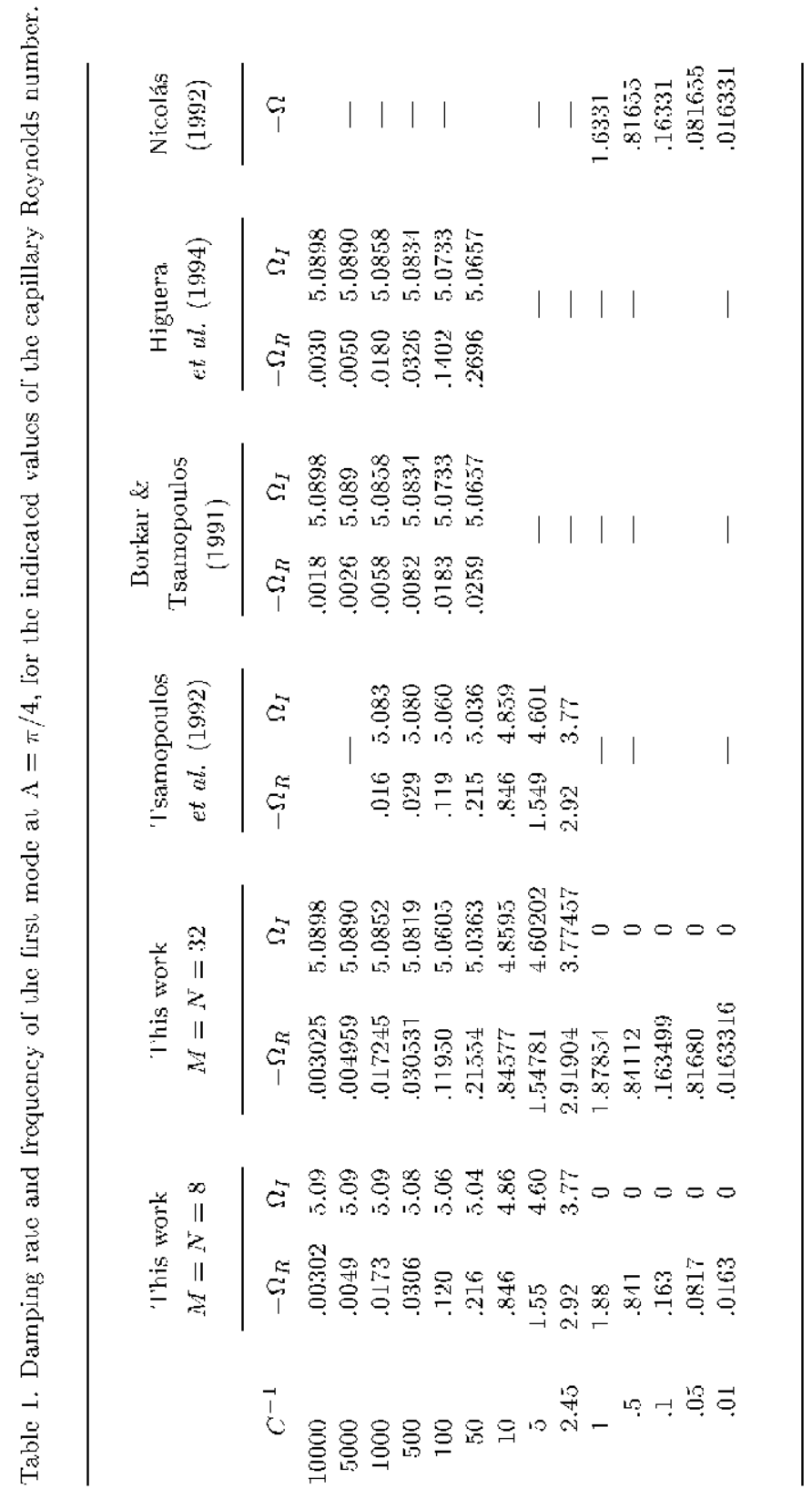


three calculated points). In this way we obtain the plots of the eigenvalues in torms of $\Lambda$ for a fixed value of $C$ that are explained below. $\Lambda$ t the moment, just note that some of the plots (e.g., that in Fig. 1b) are quite involved, with many points (which are bifurcation points of the characteristic cquation) where a pair of complex conjugate eigenvalues coalesce on the real axis. The false position method converges quite slowly near those points. In order to avoid the need of approaching too much to one such point, $\left(\Omega_{0}, \Lambda_{0}\right)$, the following asymptotic expansion of the eigenvalues

$$
\Omega-\Omega_{0} \pm D_{1}\left(\Lambda-\Lambda_{0}\right)^{1 / 2}+D_{2}\left(\Lambda-\Lambda_{0}\right)+O\left(\left|\Lambda-\Lambda_{0}\right|^{3 / 2}\right)
$$

may be used in its vicinity, where the real constants $D_{1}$ and $D_{2}$ can be numerically computed in cach case.
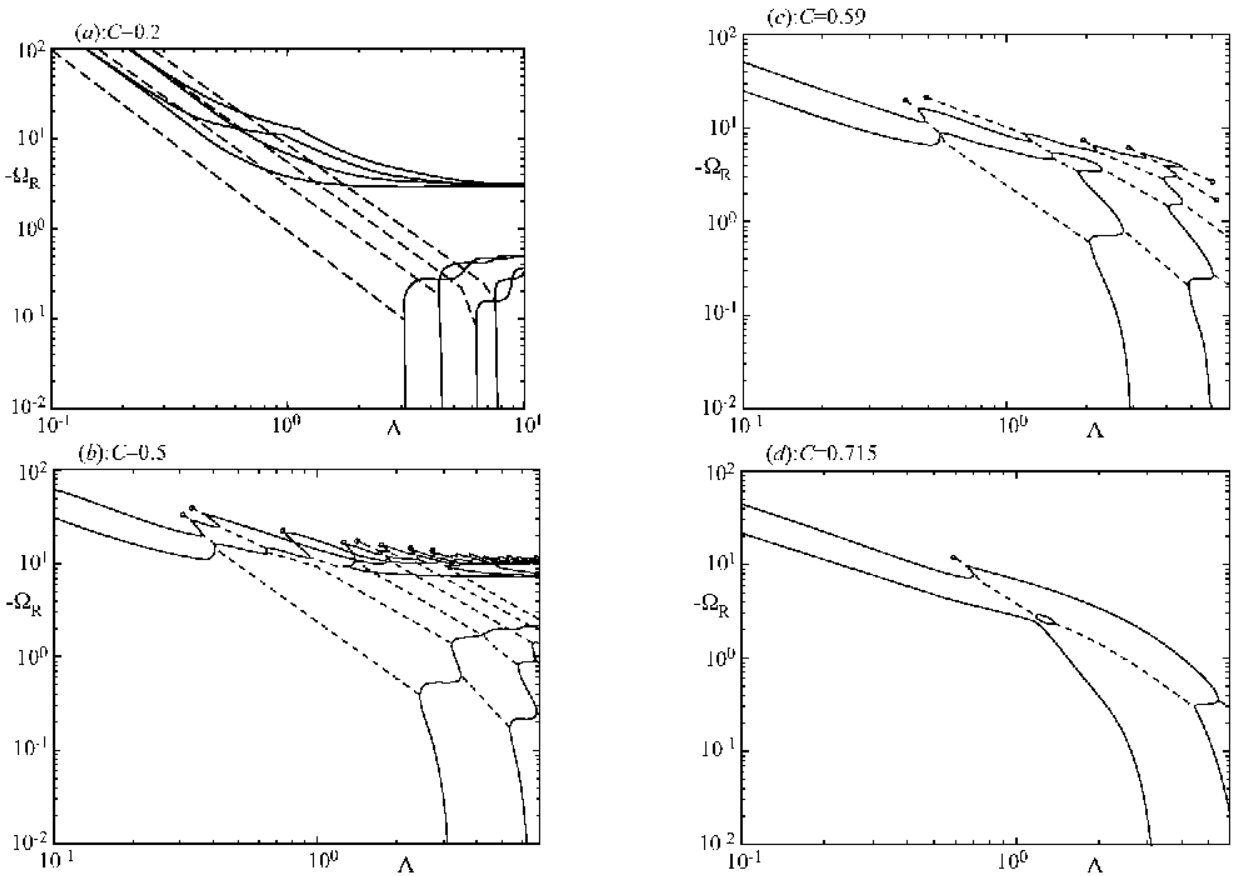

Figure 1.

The damping rate $-\Omega_{R}$ in terms of the slenderness $\Lambda$ for the indicaled values of the capillary number $C$. 'T' he first few odd and even modes are considered in plot (a), while only odd modes are considered in the remaining plots. Real and complex eigenvalues are plotted with solid and dasherl lines respectively.

The plot of the damping rate in terms of $\Lambda$ for $C-.2$ for some of the first eigenmodes, is given in Fig. 1a, where the real eigenvalues are plotted with solid lines and the complex ones with dashed lines. Note that there are two kinds of 
eigenvalues. Some of them (to be called capillary in the sequel) are complex in the plot exept as $\Lambda$ approaches the Rayleigh instability limits, which are the roots of

$$
\sin \Lambda-0 \quad \text { and } \quad \Lambda-\tan \Lambda-0,
$$

for odd and even modes respectively ( $\operatorname{then} \Lambda \simeq 3.14,1.49,6.28$ and 7.73 for the first four capillary modes). But, in addition to these capillary modes, there are other branches of (hydrodynamic) eigenvalues that are always real and were not detected in [23]. Tote that these hydrodynamic modes should not be ignored because they exhibit a damping rate that is of the same order as that of the capillary ones, and this is more true as $C$ decreases, as we shall see in $\$ 1.1$. Also, even though they do not affect much the linear evolution of the interface, they do affect the evolution of the velocity ficld, and morcover they are conceptually important because they are essentially associated with steady streaming, as it, will be explained in \$4.1.1. The main difference between capillary and hydrodynamic modes is that the free surface deformation plays an essential role in the former but is unimportant in the latter (that would also exist if the free surface were undeformable). In order to illustrate this statement, the froe surface deformation $F$ and the axial velocity at the interlace, $W-\phi_{r}+r^{-1} \phi$, are plotled in Fig. 2 for $\Lambda-1, C-.1$ and the first, capillary (plot(a)) and hydrodynamic (plot(b)) modes; note that the free surface deformation is quite small for the latter (in fact, $F(z) \sim C W(1, z)$ as $C \rightarrow 0$ ). The clear distinction between capillary and hydrodynarric modes in Fig. 1 a is due to the fact that $C$ is small (cven though for $C$ large a similar distinction appears, as it will be seen in \$4.2), $\Lambda$ is not too small and we are not ploting higher order modes. For a small but fixed value of $C$ that clear distinction disappears (and the associated branches $\Omega=\Omega(\Lambda)$ morgo) as cither $\Lambda \sim C^{2}$ for a fixed capillary mode or as $\Omega \sim C^{-3}$ for a fixed value of $\Lambda$, as it. will be seen in $\$ 4.1$; in both limits capillary modes merge with the hydrodynamic ones and the associated eigenvalue $\Omega$ becomes real. This is not apprecialed in Fig. la where (in order to better illustrate the remaining above-mentioned properties) we neither consider the interval $.01<\Lambda<.1$ nor plot higher order modes, but this effect is clearly seen in Fig. 1b, which is considered now.

The plot of $-\Omega_{k}$ vs. $\Lambda$ for smaller values of $C$ is similar to that in Fig. 1 a. (as it will be seen in \$4.1). As $C$ increases, instead, the above-mentioned region, where capillary and hydrodynamic modes merge, enlarges and finally all modes become real. 'That merging process takes place fairly quickly in the interval .55 $C \leqq 1$, that is considered now in Figs. 1b, $c$ and d where for the sake of clarity an appropriate number of odd modes are considered; even modes behave similarly. Note that since odd and even modes obey different characteristic equations, they behave independently and do not mix up. The plot in Fig. 1 b shows that the branches are quite involved in the upper morging region, where the numerical continuation process becomes increasingly costly. The lower and upper merging regions approach each other very quickly and merge as $C$ is further increased (see Figs. 1c and d) and finally, for $C \gtrsim .8$, the first two odd eigenvalues become real. 

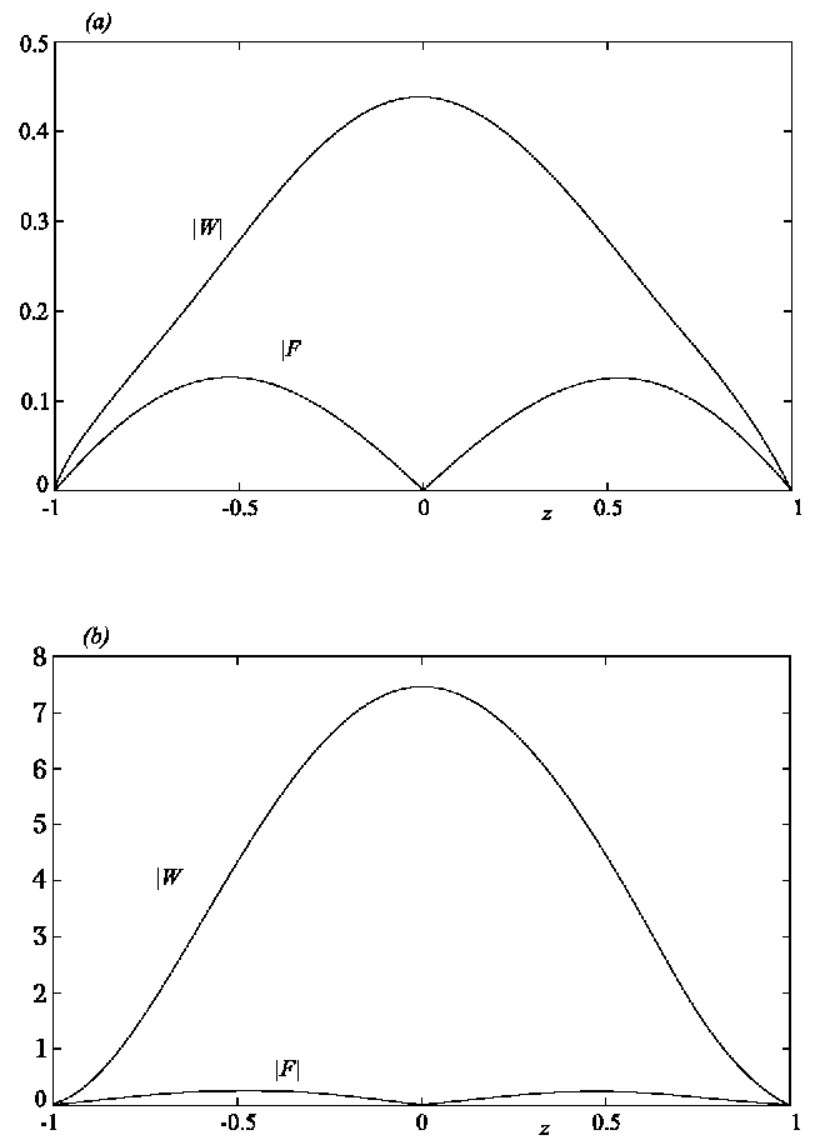

Figure 2.

The free surface deformation $|\Gamma|$ and the axial velocity at the interface $|W|$ in terms of the axial coordinate for $C-2, \Lambda-1$ and (a) the first capillary mode and (b) the first hydrodynamic mode.

'The shape of the real branches in Fig. 1e suggests the formation of isolas (as that in Fig. 1d), but they may survive only for $C$ in quite narrow intervals. Note that the distinction between capillary and hydrodynamic modes is not clear in this merging interval $5 \leqslant C \leqslant 1$; that distinction will appear again for still larger values of $C$, as we shall see in $\S 4.2$.

\section{Asymptotic results for limiting values of $C$ and $A$}

Iet us consider now the relevant limiting values of $C$ and $\Lambda$, mamely, $C \ll 1$, $C \gg 1$ and $\Lambda \ll 1$. Note that we are ignoring here the limit $\Lambda \gg 1$ because for $\Lambda>\pi$ the bridge is unstable due to the Rayleigh instability. Nevertheless, 
that limit leads to the well-known 1-D models that have been thoroughly analyzed in the literature, and claimed to provide good results for moderate (i.e, smaller than $\pi$ ) values of $\Lambda$; that claim will be discussed in $\S 5$. The aim of this section is two-fold. On the one hand, we pursue the understanding of the dependence of the frequency and damping rate on the parameters $C$ and $\Lambda$. On the other hand, we seek for simplified characteristic equations that apply in the limiting cases, and analyze the scope of the associated approximate results.

The limiting cases below could be directly analyzed in the characteristic equations derived in 33 , which are valid for arbitrary values of the parameters. But then we would lose any insight into the relevant physical mechanisms that apply in each limit. Therefore, we shall consider below the approximate asymptotic equations in each limit, whose associated characteristic equation is precisely the one obtained mathematically as the limit of the characteristic equation derived in $\S 3$.

\subsection{The nearly-inviscid limit $C \rightarrow 0$}

If $C$ is small then two distinguished limits must be considered, $|\Omega| \sim C$ and $|\Omega| \sim 1$, which yicld hydrodynamic modes and capillary modes respectively. Let us point here that hydrodynamic modes have not been al ways explicity considered (the only exceptions seem to be [20] and [21]) in the linear analysis of liquid bridges. This is so in spite of the fact that their existence in nearly-inviscid surface wave problems may be anticipated (as already pointed out by Lamb [34]) by just noticing that the limit $C \rightarrow 0$ is a singular perturbation limit. When considering capillary modes, viscous terms are ignored in first approximation; consequently, the original fourthorder equation (2.2) for the stream function becomes a second-order equation, and a part of the spectrum is necessarily lost.

IIydrodynamic modes correspond to solutions of (2.2) (2.7) such that $|\Omega| \sim$ $|F| \sim C$ if the cigenfunction is taken such that $|\phi| \sim 1$. 'Then $\Omega, F$ and $\phi$ are expanded as

$$
\Omega-C \Omega_{1}+\ldots, \quad F-C F_{1}+\ldots, \quad \phi-\phi_{0}+\ldots
$$

where $\Omega_{1}$ is real and negative and was lirst oblained by Higuera et al. $[20]$, to be

$$
\Omega_{1}--\lambda_{n}^{2}-\mu_{n m}^{2}
$$

in terms of the radial and azimuthal wavenumbers $n$ and $m$, where $\lambda_{1}, \lambda_{2}, \ldots$, are the positive roots of $J_{1}\left(\lambda_{n}\right)=0$, and, for each $n, \mu_{n 1}, \mu_{n 2}, \ldots$, are the (strictly) posilive roots of one of the following equations:

$$
\begin{aligned}
& \mu_{n m m} \tanh \left(\lambda_{n} \Lambda\right)-\lambda_{n t} \tan \left(\mu_{n m m} \lambda\right), \\
& \lambda_{n} \tanh \left(\lambda_{n} \Lambda\right)+\mu_{n m} \tan \left(\mu_{n m} \Lambda\right)-0 .
\end{aligned}
$$




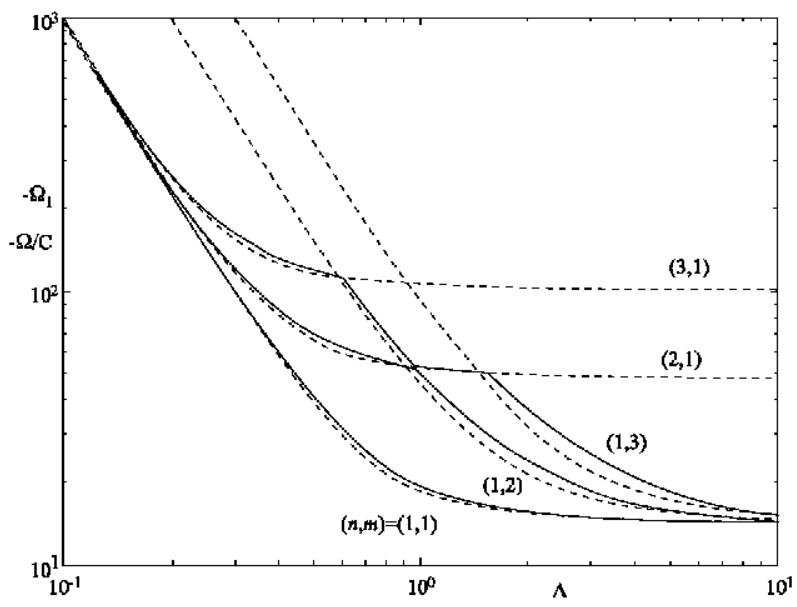

Figure 3.

Lamping rate of the hydrodynamic odd modes; (----) $-\Omega_{1}$ as calculated from (4.2) for the indicated values of $n$ and $m$, (-) $-\Omega / C$ for $c-.2$ as calculated from the characteristic equation derived in Ser. III; $n$ and $m$ are the radial and axial wavenumbers.

'The plot of $-\Omega_{1}$ in terms of the slenderness (as calculated from (1.2) for the first rew odd modes is given with dashed lines in Fig. 3, where the exsentially exact values of $-\Omega / C$ (as calculated from the characteristic equation (3.24) derived in (3) for $C=.2$ are also plotted with solid lines for comparison; the plot for cven modes is completely similar. Note that the approximation is quite good even for that not-so-small value of $C$. 'The main differenee between the exact and approximale branches is that they are connected in a diflerent. way near the double points of the approximate curves. Thus those parts of the dashed lines not followed by continuous lines just correspond to the fourth and upper branches of the exact solutions (for $C-0.2$ ), which are not given in Fig. 3. Also, in some sense, the hydrodynamic modes appear as a multiple cigenvaluc $\Omega \simeq 0$ in as standard direct numerical calculation; this could explain that these eigenvalues were not detected in [23]. Still, as anticipated above, the free surface deformalion $F$ is quile small. for hydrodynamic modes (see (1.1)). Thus these modes are expected to play a secondary role in free linear oscillations if only the free surface deformation is observed; but they do play an essential role in linear or weakly nonlincar oscillations ir, in addition, the flow pattern inside the liquid bridge is considered, as we discuss below, in $\S 4.1 .1$.

For capillary modes the cigenvalue $\Omega$ may be expanded as

$$
\Omega- \pm i \Omega_{0}-C^{1 / 2}(1 \pm i) \Omega_{1}-C \Omega_{2}+O\left(C^{3 / 2}\right)
$$

where $\Omega_{0}, \Omega_{1}$ and $\Omega_{2}$ are real and strictly positive if $0<\Lambda<\pi$. The first term corresponds to the inviscid limit and was lirst calculated by Sanc $[17]$, while the 
second term (first obtained by Borkar \& Tsamopoulos [19]) accounts for the leading order effect of the Stokes boundary layers near the disks. The third term is real and accounts for viscous dissipation in the bulk and a first correction of viscous dissipation in the Stokes boundary layer and was calculated by Higuera et al. [20]. The first two terms in (4.3) amount, to a good approximation of the frequency $[19,20]$ but a poor approximation of the damping rate except for unrealistically small values of $C$, as anticipated above and already appreciated in Table 1 . 'This is so because $\Omega_{2} / \Omega_{1}$ is always large, of the order of $10^{2}[20]$. The first three terms in (1.3) instead give a reasonably good approximation of the damping rate, as illustrated in Figure 4 for $C-10^{-3}$ and the first Cour capillary modes; this is also true for gravity-capillary waves in cylindrical containers, where a three-term approximation like that in (1.3) has solved a discrepancy of formor theorctical calculations of the damping rates with measured ones $([13][15]$ and references given therein). Lote that the damping rate of capillary modes for $C=10^{3}$ is comparable to that of hydrodynamic modes (compare Figs. 3 and 4 and also that, for $C-10^{-3},-\Omega / C$ is quite well approximated by the plots with dashed lines in Fig. 3); for smaller values of $C,-\Omega_{R} / C$ remains essentially constant for hydrodynamic modes and increases for capillary modes (see (4.1) and (4.3)).

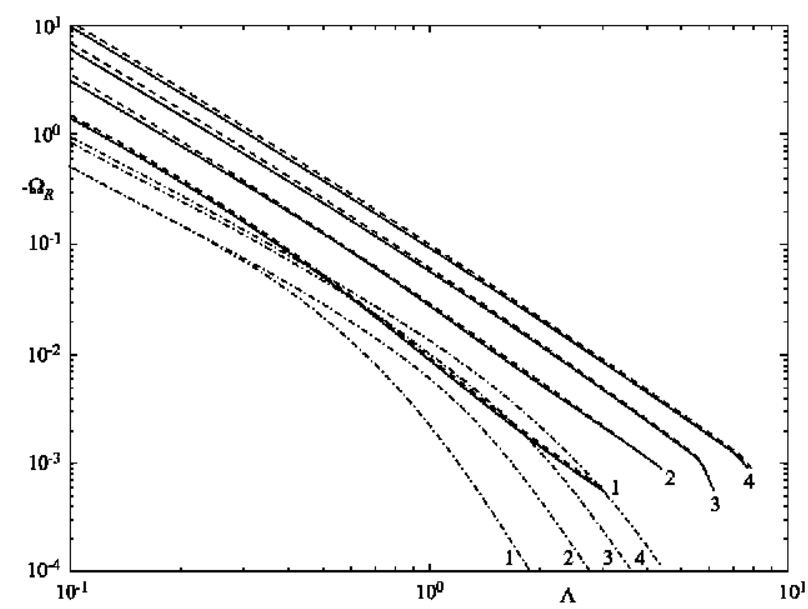

Figure 1.

Damping rate of the first four capillary modes for $C=10^{3}$ : (—) as calculated from the characteristic equalion derived in Sec. III; (---) as given by the approximation (4.3); (-. - - . -) as given by the approximation in [19].

The approximation (4.3) breaks down in three limiting cases (as either $\Lambda \sim C^{2}$, $|\Lambda-\pi| \sim C^{2}$ or $\left.\Omega \sim C^{3}\right)$ that are now considered in some detail.

a.- The size of the oscillatory boundary layers is of the order of $\left(C / \Omega_{0}\right)^{1 / 2}$. Since $\Omega_{0} \sim \Lambda^{-3 / 2}$ as $\Lambda \rightarrow 0$, when $\Lambda \sim C^{2}$ (or smaller than that) the Stokes boundary layers are no longer small as compared to the slenderness and the approximation 
above must fail. The distinguished limit now is $\Lambda \sim C^{2} \rightarrow 0, \Omega \sim \Lambda^{-3 / 2}$ and its analysis (which explains the loft part of the upper merging region in Fig. 1b) is beyond the scope of this paper. Instead, the simpler sub-limit $\Omega \sim(\Lambda C)^{-1}$, with $\Lambda \ll C^{2}$, will be considered in $\$ 1.3$, as a particular case of the limit $\Lambda \rightarrow 0$, to illustrate that the eigenvalues must be real it $\Lambda$ is sulficiently small.

b.- The frequency $\Omega_{0}$ vanishes at the positive roots of the two equations $(3.27)$. In particular, the frecuency associated with the first modo vanishes at $\Lambda=\pi$, and it is such that $\Omega_{0}^{2} /(\Lambda-\pi) \rightarrow \gamma \simeq 0.102$ as $\Lambda \rightarrow \pi$. Thus, as $\pi-\Lambda \sim C^{2}$ we have $\Omega_{0} \sim C$, the size of the oscillatory boundary layers, $\left(\Omega_{0} / C\right)^{1 / 2}$, becomes of order unity and the approximation leading to (1.3) must fail for the first mode. In order to analyze this limit for the first mode we introduce the parameter l, defined as

$$
\Lambda-\pi+l C^{2}
$$

and seek the expansions

$$
\Omega-C \Omega_{1}+\ldots, \quad \phi-C \phi_{1}+\ldots, \quad F-F_{0}+C^{2} F_{2}+\ldots .
$$

When (4.4)-(4.5) are inserted into (2.2)-(2.7), $\Omega_{1}, \phi_{1}$ and $F_{0}$ are seen to be given by a linear problem, which is written in [20] (note that there is a difference on the definition of the stream function in this paper and in [20]). The somi-analytical solution of that problem is oblained by a method like that in $\$ 3$ and is omitted for the sake of brevity. 'The plot of the real and imaginary parts of $\Omega_{1}$ in torms of $l$ is given with solid lines in Fig. 5 where, for comparison, the essentially exact, values of the real and imaginary parts of $\Omega / C$ for $C-.1$ (as calculated from the characteristic equation derived in (3) are also plotted with dashed lines. Note that, $\Omega_{1}-\Omega / C-0$ at $l-0$ (i.e., at, $\Lambda-\pi$, the Rayleight instability limit), as was to bo expected, and that the approximation above is quite good for that moderately small value of $C$ (in fact, for $C-.02$ the exact, and approximate curves are indistinguishable). Note that this limit gives that part of the branch associated with the first mode that is real near $\Lambda=\pi$ in Fig. 1a.

c.- For high-order modes the inviscid eigenfrequency $\Omega_{0}$ of the $n$-th mode grows as $n^{3 / 2}$ (as $n \rightarrow \infty$ ), and the associated stream function exhibits a fast oscillation in the axial direction, with a wave-length of the order of $n^{-1}$, and decays exponentially as $n(1-r) \rightarrow \infty$; the inviscid eigenmodes are nothing but slanding capulary wovetrains that affect only an inviseid region of thickness $n{ }^{1}$ near the free surface. If, in addition, $n C^{2} \ll 1$ then the thickness of the inviscid region is still large as compared to the thickness of the oscillatory boundary layer near the free surface, and the analysis above still applies for normal modes. If instead $n \sim C^{-2}$ (or larger than that) then the size of the oscillatory boundary layer is no longer small as compared to that of the inviscid region and the analysis above must fail. The distinguished limit, is now $|\Omega| \sim C^{-3}$ and normal modes are standing wiscous wavelrains, with a wavelength of the order of $C^{2}$, and the stream function decaying exponentially as $C^{-2}(1-r) \rightarrow \infty$. This limit corresponds precisely to 


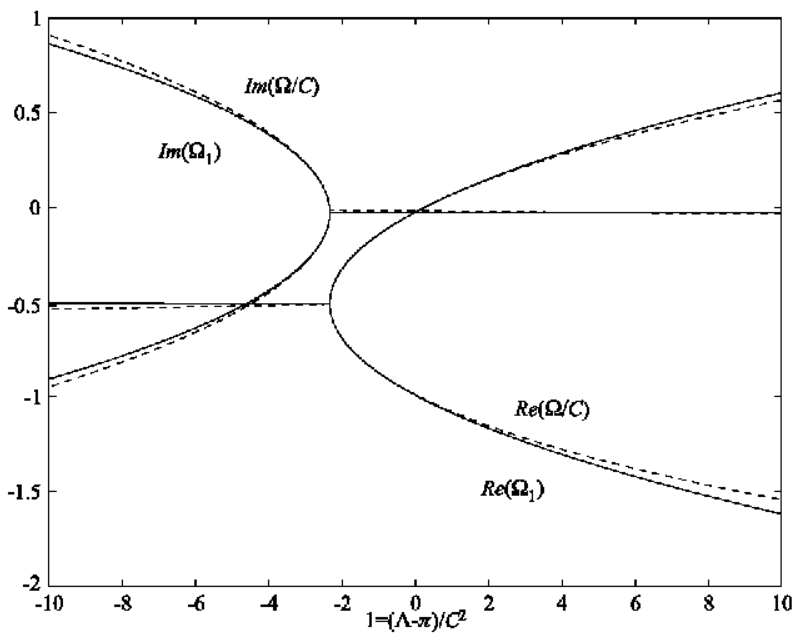

Figure 5.

The leading order coefficient $\Omega_{1}$ in (1.5) in terms of $l(-)$, and the exact value of $\Omega / C$ in terms of $(\Lambda-\pi) / C^{2}$ for $C-.1(----)$.

the upper merging region in Fig. $1 \mathrm{~b}$, and its analysis is again beyond the scope of the paper.

Implications on the weally-nonlinear response of the bridge to monochromatic, axial vibralions of the disks. Let us now assume that the disks are axially vibrated in such a way that their position is given by

$$
\pm \Lambda\left|\varepsilon \beta_{ \pm} \operatorname{cxp}(i \omega t)\right| c \kappa .
$$

where $\varepsilon$ is appropriately small and $\beta_{+}$and $\beta$ are complex parameters of order unity accounting for the re-scaled vibrating arrplitudes and phases. Jote that we are including $g$-jitter as a particular case, when $\beta_{1}-\beta_{-}$. Since bolh capillary and hydrodynamic modes are only weakly damped, both of them must be taken into account when, analyzing the weally-nonlinear response of the bridge. Capillary modes are associated with capillary waves that aro directly excited by tho vibration of the disks. Hydrodynarnic modes, instead, are associated with a secondary streaming how (which is steady if forcing is appropriately small but may be slowly varying otherwise). 'This flow is forced by woll known mochanisms resulting from paramedric resonance eflects in the oscillatory boundary layers, first considered by Schlichting [35] and Longuet-IIiggins [36]. Thus hydrodynamic modes explain the (surprising at first sight) fact that small-amplitude disks vibrations produce nonlinear streaming flows, with an associated effective Reynolds number that can be fairly large; these flows appcar in a natural way in the weakly-nonlinear doscription of the capillary bridge response. The spatio-temporal structure of both the capillary waves and the streaming flow highly depends, both qualitatively and quantitatively, on whether the forcing frequency is of order unity or large (the distinguished 
limits $\omega \sim C^{-1}$ and $\omega \sim C^{-3}$ must be considered separately when $\omega$ is large) and on whether $\omega$ is close to an inviscid natural frequeney or not if $\omega$ is of order unity; if $\omega \sim C^{-1}$ then $\omega$ is always close to many inviscid natural frequencies (because two consocutive natural frequoncios satisfy $\Omega_{n \mid 1}-\Omega_{n} \sim n^{1 / 2} \ll \Omega_{n} \cdots n^{3 / 2}$ if $\Omega_{n} \sim C^{-1}$ ) and many capillary modes mus be considered simultaneously that amount to a pair of capillary wavetrains that are forced and reflected at the disks, and are damped by viscous dissipation as they propagate along the free surface. See $[3],[8]$ and $[9]$ for the analysis of the limits $\omega \sim 1$ and $\omega \sim C^{-1}$.

\subsection{The strongly viscous limit $C \rightarrow \infty$}

Let us now consider the limit $C \rightarrow \infty$, to obtain asymptotic results that yicld good approximations for moderately large values of $Q$ (roughly, $C \geq 2$ ). We rust consider two distinguished limits, $\Omega \sim C^{-1}$ and $\Omega \sim C$, which yield capillary modes and hydrodynamic modes respectively.

For capillary modes we seek the expansions

$$
\Omega=C^{-1} \Omega_{1}\left|\ldots, \quad \phi=C^{-1} \phi_{1}\right| \ldots, \quad F=F_{0}^{\prime} \mid \ldots,
$$

where (upon substitution into (2.2)-(2.7) and setting to zero the leading orders torms) $\Omega_{1}, \phi_{1}$ and $F_{0}$ are seen to bo given by

$$
\begin{gathered}
\mathcal{L}^{2} \phi_{1}-0 \quad \text { in } 0<r<1, \quad-\Lambda<z<\Lambda \\
\phi_{1}-\phi_{1 z}-0 \text { at } z- \pm \Lambda, \quad \phi_{1}-\mathcal{L} \phi_{1}-0 \text { at } r-0 \\
\phi_{1 z}\left|\Omega_{1} F_{0}^{\prime}=\phi_{1 r r}\right| \phi_{1 r}-\phi_{1}-\phi_{1 z z}=0 \quad \text { at } r=1 \\
F_{0}^{\prime \prime \prime}\left|F_{0}^{\prime \prime}\right| \phi_{1 r r r}\left|3 \phi_{1 r z z}\right| 3 \phi_{1 r r}=0 \text { at } r=1 \\
F_{0}( \pm \Lambda)-\int_{-\Lambda}^{\Lambda} F_{0}(z) \mathrm{d} z-0 .
\end{gathered}
$$

Hore the operator $\mathcal{C}$ is as defined in (2.8). The engenoalue $\Omega_{1}$ is always real and negative, if $\Lambda<\pi$. In order to prove this property, multiply (4.7) by $\bar{\phi}_{1}$ (the complex conjugate of $\phi_{1}$ ), integrate in $0<r<1,-\Lambda<z<\Lambda$, integrate by partis repeatedly and take into account the boundary conditions (1.8)-(1.11) to obtain

$$
\begin{aligned}
& \bar{\Omega}_{\perp} \int_{-\Lambda}^{\Lambda}\left[\left|F_{0}^{\prime 2}\right|-\left|F_{0}\right|^{2}\right] \mathrm{d} z--\int_{-\Lambda}^{\Lambda} \int_{0}^{1}\left|\phi_{1 r r}+r^{1} \phi_{1 r}-r^{2} \phi_{1}-\phi_{1 z z}\right|^{2} r \mathrm{~d} r \mathrm{~d} z \\
&-2 \int_{-\Lambda}^{\Lambda} \int_{0}^{1}\left[\left|\phi_{1 r z}\right| r^{\left.\left.\left.\left.1_{\phi_{1} z}\right|^{2}|| \phi_{1 r z}\right|^{2}\left|r^{2}\right| \phi_{1 z}\right|^{2}\right] r \mathrm{~d} r \mathrm{~d} z} .\right.
\end{aligned}
$$

Thus $\Omega_{1}$ is real. If, in addition, $\Lambda<\pi$ then $\int_{\Lambda}^{\Lambda}\left(\left|F_{0}^{\prime}\right|^{2}-\left|F_{0}\right|^{2}\right) \mathrm{d} z>0$ (according to the variational definition of the first eigenvalue of $F^{\prime \prime}+F+\lambda F-0$ if $-\Lambda<$ 
$z<\Lambda, F( \pm \Lambda)-0$, which is strictly positive if $\Lambda<\pi$ ), and $\Omega_{1}$ is obviously strictly negative, as stated.

Now, the linear problem (4.7) (4.11) is solved by a semi-analytical method like that in 33 , to obtain the semi-analytical solution in the $\Lambda$ ppendix; alternatively, the semi-analytical solution may be oblained directly from that in $\$ 3$ when taking the limit suggested by the expansions (4.6). When solving (4.7) (4.11) in this way we obtain the plot of $-\Omega_{1}$ in terms of $\Lambda$ given by solid lines in Fig. 6 for the first four modes. For comparison we also give with dashed lines the essentially exact values of $-C \Omega$ for $C=2$, as calculated by the characteristic equation in \$3. Note that $\Omega_{1}-C \Omega-0$ al $\Lambda-\pi$ (the Rayleigh instability limit) as was to be expected, and that the approximation is quite good even for that not-so-large value of $C$. Let us point here that the problem (4.7)-(1.11) was first solved in Vicolás [22] by means of biorthogonal series, but that solution is much more expensive than the semianalytical solution considered here.

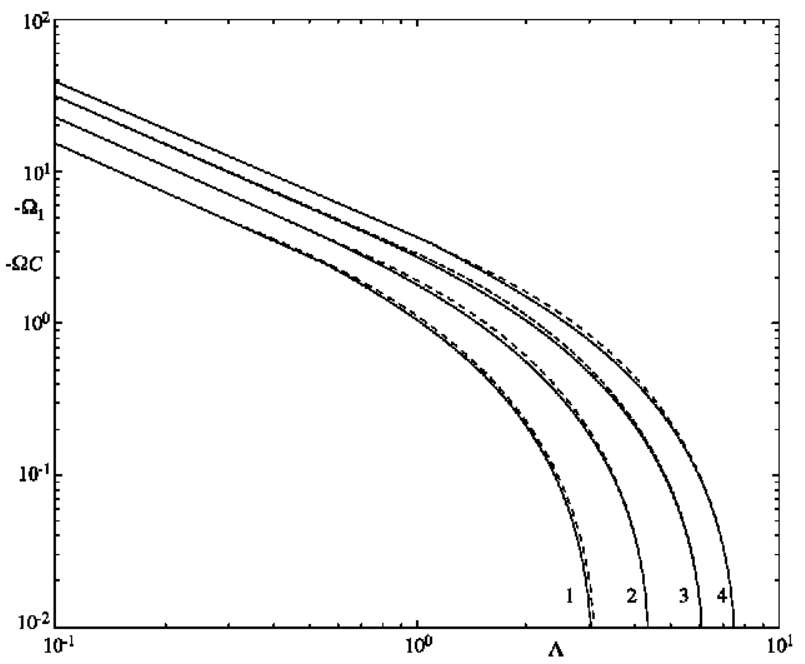

Figure 6.

'The leading order coefticient in (4.6), $-\Omega_{1}$, in terms of the slenderness (- $\longrightarrow$ ), and the exact value of $-32 C$ for $C-2(----)$, for the first four capillary modes.

For hydrodynamic modes we seek the expansions

$$
\Omega-C \Omega_{0}+\ldots, \phi-\phi_{0}+\ldots, \quad F-C^{-1} \Gamma_{1}+\ldots,
$$

where $\Omega_{0}$ and $\phi_{0}$ are given by

$$
\begin{aligned}
& \left(\mathcal{L}-\Omega_{0}\right) \mathcal{L} \phi_{0}-0 \quad \text { in } 0<r<1, \quad-\Lambda<z<\Lambda, \\
& \phi_{0}-\phi_{0 z}-0 \text { at } z- \pm \Lambda, \quad \phi_{0}-\mathcal{L} \phi_{0}-0 \text { at } r-0 \\
& \phi_{0 r r} \mid \phi_{0 r}-\phi_{0}-\phi_{0 z z}=\Omega_{0}\left(\phi_{0 r} \mid \phi_{0}\right) \\
& \quad-\left(\phi_{0 r r r}+3 \phi_{0 r z z}+3 \phi_{0 r r}\right)-0 \quad \text { at. } r-1
\end{aligned}
$$


while $F_{1}$ is seen to be as given by $F_{1}--\phi_{0 z} / \Omega_{0}$. IIere the operator $\mathcal{L}$ is again as defined in (2.8). The cigenvalue $\Omega_{0}$ is always real and negative, as readily seen from the following equation

$$
\begin{aligned}
& \Omega_{0} \int_{\Lambda}^{\Lambda} \int_{0}^{1}\left(\left|\phi_{0 r}+r^{1}{ }^{1} \phi_{0}\right|^{2}+\left|\phi_{0 z}\right|^{2}\right) r \mathrm{~d} r \mathrm{~d} z \\
& --\int_{-\Lambda}^{\Lambda} \int_{0}^{1}\left[\left|\mathcal{L} \phi_{0}-\phi_{0 z z}\right|^{2}+2\left|\phi_{0 r z}+r{ }^{1} \phi_{\phi_{0 z}}\right|^{2}+2\left|\phi_{0 r z}\right|^{2}+2 r^{2}\left|\phi_{0 z}\right|^{2}\right] r \mathrm{~d} r \mathrm{~d} z
\end{aligned}
$$

which is readily obtained upon multiplication of $(4.13)$ by $\bar{\phi}_{0}$, integration in $0<r<1,-\Lambda<z<\Lambda$, integration by parts and substitution of the boundary condilions (4.14)-(4.15). The linear problem (4.13)-(4.15) is solved by a semianalytical method like that in $\S 3$. For the sake of brevity that semi-analytical solution of (4.13)-(4.15) is omitted. The plot of $-\Omega_{0}$ in terms of $\Lambda$ for the first lew odd modes is found to be as that, in Fig. 7, where again we have also plotted with dashed lines the essentially exact values of $-\Omega / C$ for $O=5$. lote that again the approximation is quite good. Also, contrary to Fig. 3, there are no intersections between different approximate branches and the exact and approximate branches are connected in a completely similar way. This has becn carefully checked, specially for higher order modes, near those points that behave as double points at first sight.

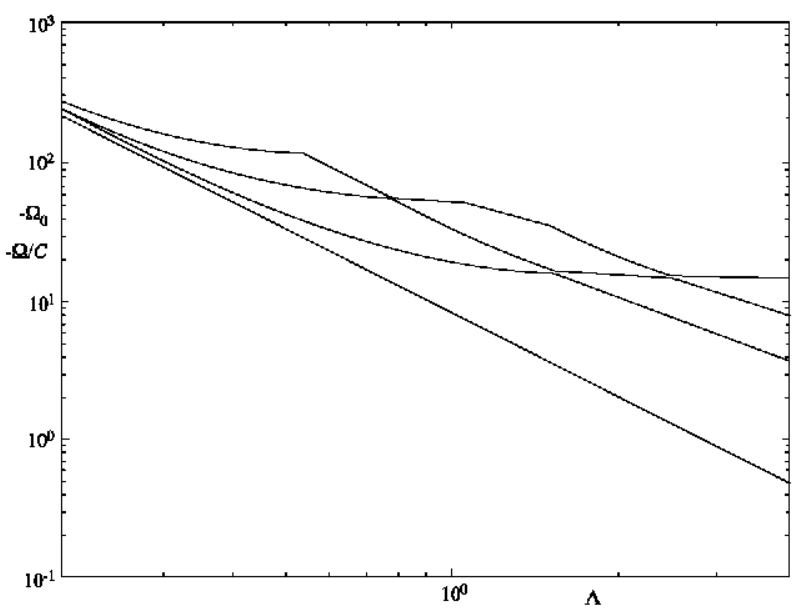

Figure 7 .

The leading order coefficient in (4.12), $-\Omega_{0}$, in terms of the slenderness (- $\longrightarrow$, and the exact values of $-\Omega / C$ for $C-5(---)$, for the first four odd hydrodynamic modes (both curves are almost indistinguishable).

A comment on these two kinds of modes is now in order. As it happened when $C \ll 1$, the main difference between capillary and hydrodynamic modes is that 
the free surface deformation is essential in the former and it plays no role in the latter (sec (4.6) and (1.12)). But now both kinds of modes are always overdamped (i.e., $\Omega$ is real) and the spectra is well separated (damping is much higher for hydrodynamic modes than for capillary ones, and thus the former have no much interest in practice).

\subsection{Short liquid bridges: $\Lambda \rightarrow 0$}

If $C$ is kept constant (and of order unity) then as $\Lambda \rightarrow 0$ wo must distinguish two asymptotic limits, $|\Omega| \sim \Lambda^{-1}$ and $|\Omega| \sim \Lambda^{-2}$, that correspond to capillary modes and hydrodynamic modes respectively. The analysis of the latter is beyond the scope of this paper; their associated eigenfunctions exhibit fast oscillations (with a wavelength of the order of $\Lambda$ ) in the radial direction, and their analysis requires to consider three distinguished regions, $r \sim \Lambda, \Lambda \ll r \sim 1-r$ and $1-r \sim \Lambda$.

Capillary modes are calculated now by considering the stretched variables

$$
\eta=z / \Lambda \quad \text { and } \quad \xi=(r-1) / \Lambda
$$

and introducing the expansions

$$
\Omega-(\Lambda C)^{1}\left(\Omega_{0}+\ldots\right), \quad \phi-\phi_{0}+\ldots, \quad F-C F_{0}+\ldots
$$

When (4.16) (4.17) are inserted into (2.2)-(2.7) and the leading order terms are sel to zero, the following problem results

$$
\begin{aligned}
& \left(\partial^{2} / \partial \xi^{2}+\partial^{2} / \partial \eta^{2}\right)^{2} \phi_{0}-0 \quad \text { in }-\infty<\xi<0, \quad-1<\eta<1 \\
& \phi_{0}=\phi_{0 \eta}=0 \quad \text { at } \eta= \pm 1, \quad \phi_{0} \rightarrow 0 \quad \text { as } \xi \rightarrow-\infty \\
& \phi_{0 \eta}+\Omega_{0} F_{0}-\phi_{0 \xi \xi}-\phi_{0 \eta \eta}-F_{0}^{\prime \prime \prime}+\phi_{0 \xi \xi \xi}+3 \phi_{0 \xi \eta \eta}-0 \quad \text { al. } \xi-0 \\
& F_{0}( \pm 1)-\int_{-1}^{1} F_{0}(\eta) \mathrm{d} \eta-0 .
\end{aligned}
$$

Some remarks about this model are now in order:

a.- The problem $(4.18)-(4.21)$ provides the eigenvalue $\Omega_{0}$, which is real and negative as we prove now. To this end, first note that the asymptotic behavior, as $\xi \rightarrow-\infty$, of the non-trivial solutions of (1.18)-(1.21) is given by one of the following expressions

$$
\begin{aligned}
& \phi_{0}=K\left[\cos \delta_{1} \sin \left(\delta_{1} \eta\right)-\eta \sin \delta_{1} \cos \left(\delta_{1} \eta\right)\right] \operatorname{cxp}\left(\delta_{1} \xi\right)|c c .| \text { HO' } \\
& \phi_{0}-K\left[\sin \delta_{2} \cos \left(\delta_{2} \eta\right)-\eta \cos \delta_{2} \sin \left(\delta_{2} \eta\right)\right] \exp \left(\delta_{2} \xi\right)+c . c .+ \text { HOT }
\end{aligned}
$$

where $K+0$ is a complex constant, IIOT stands for higher order terms and $\delta_{1}$ and $\delta_{2}$ are the first-quadrant complex roots of $\sin \left(2 \delta_{1}\right)-2 \delta_{1}$ and $\sin \left(2 \delta_{2}\right)+2 \delta_{2}-0$. 
Then $\phi_{0}$ and their partial derivatives decay exponentially as $\xi \rightarrow-\infty$ and the manipulations with the integrals below are justified. Now we only need to multiply (4.18) by $\bar{\phi}_{0}$ (the complex conjugate of $\phi_{0}$ ), integrate the resulting equation in $-\infty<\xi<0,-1<\eta<1$, integrate by parts repeatedly and apply the boundary conditions (4.19)-(4.21) to obtain

$$
\bar{\Omega}_{0} \int_{1}^{1}\left|\Gamma_{0}^{\prime}(\eta)\right|^{2} \mathrm{~d} \eta--\int_{1}^{1} \int_{\infty}^{0}\left[\left|\dot{\phi}_{0 \xi \xi}-\phi_{0 \eta_{\eta}}\right|^{2}+4\left|\phi_{0 \xi \eta}\right|^{2}\right] \mathrm{d} \xi \mathrm{d} \eta
$$

Thus $\Omega_{0}$ is real and negative, as stated.

b.- The problem (1.18)-(1.21) is a canonical problem that yiclds the capillary modes of a semi-infinite liquid slab between two semi-infinite parallel plates in the limit when the capillary Reynolds number (based on the distance between the plates) is small.

c.- Although we assumed that the capillary Reynolds number $C$ is of order unity, the model (1.18)-(1.21) applies whenever $\Lambda \mid \Lambda / C^{2} \ll 1$ for arbitrary values of $C$, as is readily seen. Therefore the solution to that problem also gives the asymptotic: behavior of the solution of the model (4.7)-(1.11), considered in $\$ 1.2$, as $\Lambda \rightarrow 0$, and also the asymptotic behavior of the solution of (2.2)-(2.7) as $\Lambda \ll C^{2} \ll 1$ (and $|\Omega| \sim(\Lambda C)^{-1}$ ), as mentioned in $\S 4.1$.

d.- 'The problem (1.18)-(4.21) may be solved by a semi-analytical mothod like that in $\$ 3$, the main difference being that now we must use sine- and cosine-Fourier transform in the \& variable; alternatively, we may take the appropriate limit, according to (4.17), in the semi-analylical solution in \$3, or, according to Remark

(c) above, we may take the appropriate limit in the semi-analytical solution of (4.7)-(1.11) which is given in the Appendix. For the sake of brevity, that semianalytical solution of (4.18)-(4.21) is omitted. When using it, the first, four (two odd and two oven) cigenvalues of (4.18)-(4.21) are found to be

$$
\Omega_{0} \simeq-1.493, \quad \Omega_{0} \simeq-2.188, \quad \Omega_{0} \simeq-3.050, \quad \text { and } \quad \Omega_{0} \simeq-3.794 .
$$

In order to check numerically the approximation above, and to illustrate the main argument in Remark (c) above, we plot in Fig. 8 the real part of $-\Omega C \Lambda$, with $\Lambda$ small, for the first mode and several representative values of $C$, from fairly small to large values.

\section{The validity of one-dimensional models}

These models are obtained in the limit $\Lambda \rightarrow \infty$. Since $\Lambda$ should not be larger than $\pi$ to avoid the Rayleigh instability, not much relcvant information should be expected from these models. Nevertheless, they provide a good approximation for the first few capillary modes and moderately large (smaller than $\pi$ ) values of $\Lambda$. For $C$ small and large this assertion has been seen to be true in [31], where 


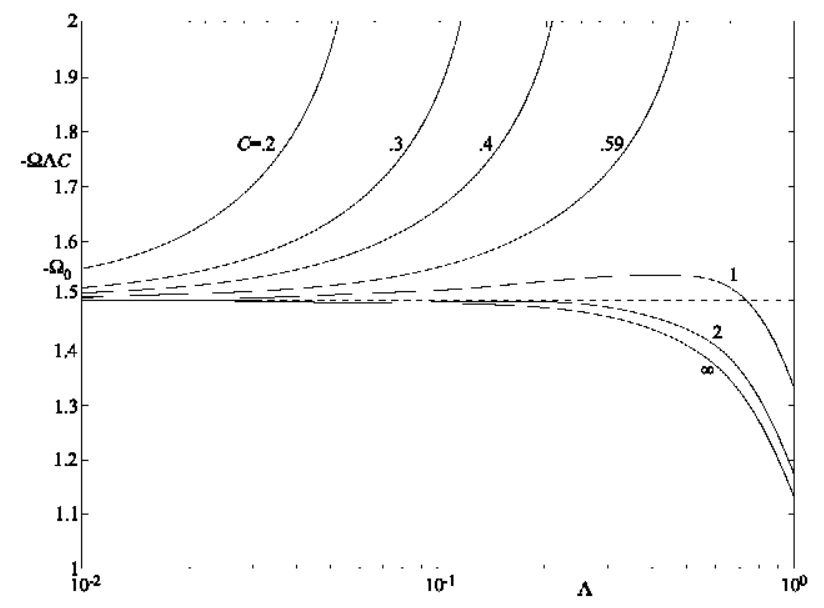

Higure 8.

$-\Omega \wedge C$ in terms of $A$ for the first mode and the indicated values of $C(\longrightarrow)$ and $-\Omega_{0} \simeq 1.493$ $(----)$.

the results oblained by these models were seen to compare well with asymptotic: results for capillary modes in [20] and in [22] (and these results provide good approximations, as wo have soen in this paper); hydrodynamic modes, instead, were not compared for large and small $C$. A comparison for an intermediale value of $C$ is given in Fig. 9, where it is seen that, again, the 1-D approximation is quite good for the first capillary mode but that, it is quite poor for the first hydrodynamic: mode even for $\Lambda-\bar{j}$ that is fairly large. Note that the approximation in $\$ 4.1$, instead, is quite good for the first hydrodynamic mode oven though $C$ is not so smatl, and this explains the failure of 1-T) results. Namely, the stream function is far from being either linear, quadratic or cubic with $r$, as assumed in 1-D models (sec [31]); according to [20] the stream function behaves radially as $J_{1}\left(\lambda_{1} r\right)$, with $\lambda_{1} \simeq 3.8317$.

\section{Concluding remarks}

We have analyzed the frec, linear, axisymmetric oscillations of a liquid bridge for varying values of the relevant parameters, the capillary Reynolds number $C^{-1}$ and the slenderness $\Lambda$. A semi-analytical method, combined with a numerical contimuation technique, has been used that provides accurate and cheap results except, of course, when two branches of the spectra approach each other (and the continuation becomes almost degenerate). Several relevant limits wero considerod and the associated asymptotic results were obtained and compared with the exact, ones. Finally, the approximate results obtained by one-dimensional models have been checked. The main conclusions are now summarized for convenience. 

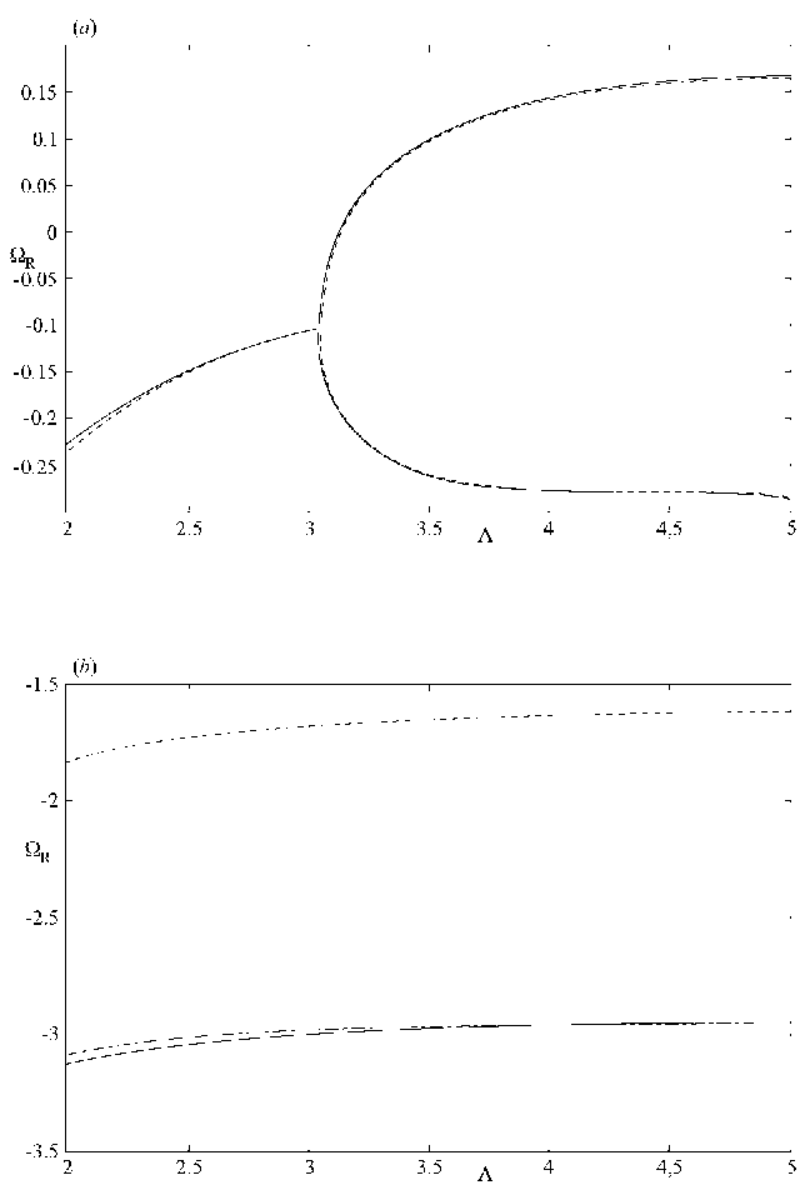

Higure 9.

The real part of the eigenvalue $\Omega$ of (a) the first capillary mode and (b) the first hydrodynamic mode in terms of the slenderness for $C=.2$. Comparison between the exact values (the approximation in [31]. (----) and the approximation (4.1) (-. -. - -).

a.- If $C$ is small two kinds of modes have been obtained. The deformation of the interface plays an essential role only in the capillary modes, which are oscillatory while the hydrodynamic modes are overdamped and were not detected in previous numerical calculations of the linear spectrum; these modes has been explained (in \$4.1.1) to be essentially related to the secondary streaming flow that is receiving an increasing attention in the literature. The semi-analytical, threetorms approximation obtained in [20] for capillary modes has becn checked to provide reasontably good results for $C \lesssim .01$; a lirst, approximation (in closed-form) of the hydrodynamic modes has been also seen to provide good results for $C \leqslant .1$. The branches of capillary and hydrodynamic modes become connected with each 
other in an upper merging region (for $\Omega \sim C^{-3}$ and $\Lambda \gtrsim C^{2}$ ) and in a lower merging region (for $\Lambda \geq \pi$ ). For $\Lambda \ll C^{2}$ (or $|\Omega| \gg C^{-3}$ ) the distinction betwen capillary and hydrodynamic modes re-appear again, but now all of them are overdamped. The limit $\Lambda \ll C^{2}$ was considered in 81.3 , but the limit $|\Omega| \gg Q^{3}$ has not been treated in the paper. In fact, (even) the (linear) response of the licquid bridge to high-frequency vibration of the disks involves many normal modes and is better analyzed in terms of highly oscillatory wavetrains (sec, o.g., [9]).

b.- The structure of the spectra described above qualitatively applies whenever $C \leqslant .5$. In the interval $5 \leqslant C \leqslant 1$ there is a sharp and involved transition in the lower part of the spectra, where capillary and hydrodynamic modes coalesce and all of them become increasingly overdamped as $C$ increases. If either $\Lambda \ll 1$ or $|\Omega| \gg 1$ the distinction between capillary and hydrodynamic modes stands, with all of them overdamped and the spectra well separated (i.e., damping is much higher for hydrodynamic modes than for the capillary ones); again, no attention has been paid to the limil $|\Omega| \gg 1$. Finally, for $C \geq 2$ the asymptotic results for $C \gg 1$ apply; the distinction between both kinds of modes re-appear everywhere, with the spectra well separated and all modes overdamped.

c- The comparison in $\$ 5$ with the approximate results from one-dimensional models has confirmed that the first fow capillary modes are woll approximated if $\Lambda \gtrsim 2$, but the hydrodynamic ones are not, and a reason for the failure has been given.

Finally, we have neglected gravity and any departure of the liquid bridge volume from the cylindrical one, and these eflects cannot be always ignored in millimetric: liquid bridges. In fact, a first approximation of these effects could have been included in a somewhat straight forward manner (along with other related weak effects such as slow rotation around the axis of symmetry). The general case, when the effects are not weak, requires of course a direct numerical calculation of the associaled linear eigenvalue problem but, even for that case, our results indicale the kind of (somewhat unexpectable) phonomena that will be oncountered, and could be missed (as it happened in [23]) if not known in advance.

\section{Appendix}

- Some expressions appearing in $\S 3$

$$
\begin{aligned}
& A_{k m}^{1}-\frac{2 \Omega\left(\lambda_{k}^{2}+2 l_{m}^{2}+\mu_{m}^{2}\right) A_{m n}^{2}}{\left(l_{m}^{2} \mid \lambda_{k}^{2}\right)\left(\nu_{k}^{2} \mid l_{m}^{2}\right)}, \quad A_{m}^{2}-\frac{l_{m} I_{1}\left(l_{m}\right)}{C\left(l_{m}^{2} \mid \mu_{m}^{2}\right) I_{0}\left(l_{m}\right)}, \\
& A_{m}^{3}-\Omega+\frac{2 C l_{m}^{2} I_{1}\left(l_{m}\right)}{I_{0}\left(l_{m}\right)}\left[\frac{I_{1}^{\prime}\left(l_{m}\right)}{I_{1}\left(l_{m}\right)}-\frac{2 l_{m} \mu_{m}}{l_{m}^{2} \mid \mu_{m}^{2}} \frac{I_{1}^{\prime}\left(\mu_{m}\right)}{I_{1}\left(\mu_{m}\right)}\right] \\
& A_{n}^{4}-\frac{2 C \lambda_{n}^{2}-\Omega}{\lambda_{n}^{2}+1}, \quad A_{n}^{5}-\frac{2 C \lambda_{n} \nu_{n}}{\nu_{n}^{2}+1}, \quad A_{n}^{6}-\frac{\Omega\left(1+\nu_{n}^{2}-2 \lambda_{n}^{2}\right)}{\lambda_{n}\left(\lambda_{n}^{2}+1\right)\left(\nu_{n}^{2}+1\right) \Lambda},
\end{aligned}
$$




$$
\Lambda_{m n}^{7}=\frac{2 \Omega\left(1-l_{m}^{2}\right)\left[\nu_{n}^{2}\left(\mu_{m}^{2}-\lambda_{n}^{2}+1\right)+3 l_{m}^{2}\right\rfloor \lambda_{n}}{\Lambda\left(\nu_{n}^{2}+1\right)\left(\lambda_{n}^{2}+1\right)\left(\nu_{n}^{2}+l_{m}^{2}\right)\left(\lambda_{n}^{2}+l_{m}^{2}\right)}, \quad \Lambda_{n}^{8}=\frac{\Omega\left(\nu_{n}^{2}+3\right) \lambda_{n}}{\left(\nu_{n}^{2}+1\right)\left(\lambda_{n}^{2}+1\right)}
$$

- Semi-analylical solution lo $(4.7)-(4.11)$. $\phi_{1}$ and $F_{0}$ are given by

$$
\begin{aligned}
& \phi_{1}- \frac{\Omega_{1}}{2} \sum_{m=1}^{\infty}\left[\left(1+\frac{l_{m} I_{1}^{\prime}\left(l_{m}\right)}{I_{1}\left(l_{m}\right)}\right) \frac{I_{1}\left(l_{m} r\right)}{I_{0}\left(l_{m}\right)}-\frac{l_{m} r I_{1}^{\prime}\left(l_{m} r\right)}{I_{0}\left(l_{m}\right)}\right] \frac{\alpha_{m} \sin \left[l_{m}(z+\Lambda)\right]}{l_{m}^{2}} \\
&+\frac{\Omega_{1}}{2} \sum_{n=1}^{\infty} \frac{z \sinh \left(\lambda_{n} z\right)-\Lambda \tanh \left(\lambda_{n} \Lambda\right) \cosh \left(\lambda_{n z} z\right)}{\lambda_{n} \cosh \left(\lambda_{n} \Lambda\right)} \frac{\gamma_{n} J_{1}\left(\lambda_{n} r\right)}{J_{0}\left(\lambda_{n}\right)} \\
&+\frac{\Omega_{1}}{2} \sum_{n-1}^{\infty} \frac{z \cosh \left(\lambda_{n} z\right)-\Lambda \operatorname{coth}\left(\lambda_{n} \Lambda\right) \sinh \left(\lambda_{n} z\right)}{\lambda_{n} \sinh \left(\lambda_{n} \Lambda\right)} \frac{\delta_{n} J_{1}\left(\lambda_{n} r\right)}{J_{0}\left(\lambda_{n}\right)} \\
& F_{0}- k_{1} \sin z+k_{2} \cos z+k_{3} \\
& \mid \Omega_{1} \sum_{m-1}^{\infty}\left[\frac{l_{m} I_{0}\left(l_{m}\right)}{I_{1}\left(l_{m}\right)}-\frac{\left(l_{m}^{2}+1\right) I_{1}\left(l_{m}\right)}{l_{m} I_{0}\left(l_{m}\right)}\right] \frac{\alpha_{m} \cos \left[l_{m}(z+\Lambda)\right]}{l_{m}^{2}-1} \\
&+\Omega_{1} \sum_{m=1}^{\infty}\left[\left(\frac{\lambda_{n} \Lambda \tanh \left(\lambda_{n} \Lambda\right)}{\lambda_{n}^{2}+1}-\frac{2}{\left(\lambda_{n}^{2}+1\right)^{2}}\right) \frac{\sinh \left(\lambda_{n} z\right)}{\cosh \left(\lambda_{n} \Lambda\right)}-\frac{\lambda_{n} z \cosh \left(\lambda_{n} z\right)}{\left(\lambda_{n}^{2}+1\right) \cosh \left(\lambda_{n} \Lambda\right)}\right] \gamma_{n} \\
&+\Omega_{1} \sum_{m=1}^{\infty}\left[\left(\frac{\lambda_{n} \Lambda \operatorname{coth}\left(\lambda_{n} \Lambda\right)}{\lambda_{n}^{2} \mid 1}-\frac{2}{\left(\lambda_{n}^{2} \mid 1\right)^{2}}\right) \frac{\cosh \left(\lambda_{n} z\right)}{\sinh \left(\lambda_{n} \Lambda\right)}-\frac{\lambda_{n z} z \sinh \left(\lambda_{n} z\right)}{\left(\lambda_{n}^{2} \mid 1\right) \cosh \left(\lambda_{n} \Lambda\right)}\right] \delta_{n},
\end{aligned}
$$

where $l_{m}$ and $\lambda_{n}$ are as given by (3.3)-(3.4), while the coefficients $\alpha_{m}, \gamma_{n}, \delta_{n}, k_{1}$, $k_{2}$ and $k_{3}$ are given by

$$
\begin{aligned}
& \left.\left(\Omega_{1} B_{m}^{1}+B_{m}^{2}\right)\right) \alpha_{m}-2 k_{1} \Lambda{ }^{1} \cos \Lambda+2 \Omega_{1} \sum_{n=1}^{\infty} B_{m n}^{3} \gamma_{n_{k}} \quad \text { ir } m \text { is odd } \\
& \left.\left(\Omega_{1} B_{m}^{1}+B_{m}^{2}\right)\right) \alpha_{m}-2 k_{33} \Lambda{ }^{1} \sin \Lambda-2 \Omega_{1} \sum_{n=1}^{\infty} B_{m n}^{3} \delta_{n} \quad \text { if } m \text { is even, } \\
& {\left[\tanh \left(\lambda_{n} \Lambda\right) \mid \lambda_{n} \Lambda / \cosh ^{2}\left(\lambda_{n} \Lambda\right)\right] \gamma_{n}=-2 \sum_{m \text { odd }} B_{m, n}^{4} \alpha_{m}} \\
& {\left[\operatorname{coth}\left(\lambda_{n} \Lambda\right)-\lambda_{n} \Lambda / \sinh ^{2}\left(\lambda_{n} \Lambda\right)\right] \delta_{n}=2 \sum_{m \text { even }} B_{m n}^{4} \alpha_{m}} \\
& k_{1} \sin \Lambda-\Omega_{1} \sum_{m \text { odd }}\left[B_{m}^{1} \alpha_{m} /\left(l_{m}^{2}-1\right)-a / l_{m}^{3}\right]+(2 \Lambda / \pi)^{3} A a \Omega_{1}+\Omega_{1} \sum_{n=1}^{\infty} B_{n}^{5} \gamma_{n}, \\
& k_{2}\left(\cos \Lambda-\Lambda^{-1} \sin \Lambda\right)=-\Omega_{1} \sum_{m \text { cvon }}\left[H_{m}^{1} \alpha_{m} /\left(l_{m}^{2}-1\right)-b / l_{m}^{3}\right]-(2 \Lambda / \pi)^{3} B b \Omega_{1}
\end{aligned}
$$




$$
+\Omega_{1} \sum_{n-1}^{\infty} B_{n}^{6} \delta_{n}
$$

$k_{3} \Lambda \mid k_{2} \sin \Lambda=-\Omega_{1} \sum_{n=1}^{\infty} B_{n}^{7} \delta_{n}$

Here $A$ and $B$ are as given in (3.23), $a$ and $b$ are

$$
\begin{aligned}
& a=4 k_{1} \Lambda^{-1} \cos \Lambda-4 \Lambda^{-1} \Omega_{1} \sum_{n=1}^{\infty} B_{n}^{8} \gamma_{n}, \\
& b-4 k_{2} \Lambda{ }^{1} \sin \Lambda+4 \Lambda^{1} \Omega_{\Omega_{1}} \sum_{n=1}^{\infty} B_{n}^{8} \delta_{n},
\end{aligned}
$$

and the coefficients $B_{m}^{1}, B_{m}^{2}, B_{m n}^{3}, B_{m m}^{4}, B_{m}^{5}, \ldots, B_{n}^{8}$ are given by

$$
\begin{aligned}
& B_{m}^{1}=\frac{l_{m} I_{0}\left(l_{m}\right)}{I_{1}\left(l_{m}\right)}-\frac{\left(l_{m}^{2}+1\right) I_{1}\left(l_{m}\right)}{l_{m} I_{0}\left(l_{m}\right)}, \quad B_{m}^{2}=\frac{\left(l_{m}^{2}-1\right) I_{1}\left(l_{m}\right)}{2 l_{m} I_{0}\left(l_{m}\right)}, \\
& B_{m n}^{3}=\frac{\left[\left(l_{m}^{2}+1\right) \lambda_{n}^{2}+3 l_{m}^{2}-\lambda_{n}^{1}\right\rfloor\left(1-l_{m}^{2}\right) \lambda_{n}}{\Lambda\left(\lambda_{n}^{2}+1\right)^{2}\left(\lambda_{n}^{2}+l_{m}^{2}\right)^{2}}, \\
& B_{m n}^{4}=\frac{\lambda_{n}^{2}\left(3 l_{m}^{2}+\lambda_{n}^{2}\right) I_{1}\left(l_{m}\right)}{l_{m n}\left(l_{m}^{2}+\lambda_{n}^{2}\right)^{2} I_{0}\left(l_{m}\right)}, \quad B_{n}^{5}=\frac{2 \operatorname{la} \sinh \left(\lambda_{n} \Lambda\right)}{\left(\lambda_{n}^{2}+1\right)^{2}} \mid \frac{\lambda_{n} \Lambda}{\left(\lambda_{n}^{2} \mid 1\right) \cosh ^{2}\left(\lambda_{n} \Lambda\right)}, \\
& B_{n}^{6}-\frac{2 \operatorname{coth}\left(\lambda_{n} \Lambda\right)}{\left(\lambda_{n}^{2}+1\right)^{2}}-\frac{\lambda_{n} \Lambda}{\left(\lambda_{n}^{2}+1\right) \sinh ^{2}\left(\lambda_{n} \Lambda\right)}+B_{n}^{7}, \\
& B_{n}^{7}=\frac{\lambda_{n}^{2}-1}{\Lambda \lambda_{n}\left(\lambda_{n}^{2}+1\right)^{2}}, \quad B_{n}^{8}=\frac{\lambda_{n}\left(\lambda_{n}^{2}+3\right)}{\left(\lambda_{n}^{2}+1\right)^{2}} .
\end{aligned}
$$

Now, after truncation, the approximate characteristic equation for odd modes (resp., for even modes), giving $\Omega_{1}$ in terms of $\Lambda$, is obtained when solving $(\Lambda .5)$, (A.7) (resp., (A.6), (A.8)) to obtain $\alpha_{m}$ and $\gamma_{n}$ (resp., $\alpha_{m}$ and $\delta_{n}$ ) in terms of $k_{1}$ (resp., $k_{2}$ ), replacing the resulting expressions and (A.11) (resp., (A.12) into (A.9) (resp., (A.10)), and requiring the resulting equation to possess a non-trivial solution $k_{1}+0$ (resp., $k_{2}+0$ ). Note that, as in $\$ 3, \alpha_{m}-0$ ) for $m$ even and $\delta_{n t}-0$ for all $n$ (resp., $\alpha_{m}=0$ for $m$ oven and $\gamma_{n}=0$ for all $n$ ) when considering odd (resp., even) modes.

\section{References}

[1] D. II. Peregrine, G. Shoker; and $\Lambda$. Symon, The bifurcalion of liquid bridges. J. Fluid Mech. $212(1990), 25-39$.

[2] S. Gaudet, G. H. Mckinley and H. A. Stone, Extensional deformation of Vewtoniam liquid bridges. Phys. Fluids $8(1990), 2568-2579$. 
[3] J. A. Nicolás and J. M. Vega, Woakly nonlinear oscillations of axisymmetric liquid bridges. d. Finid Mech. $328(1096), 95-100$.

[4] 'I'. Y. Chen and J. 'l'samopoulos, Nonlinear dynamics of capillary bridges: theory. J. Fituid Mecti. 255 (1993), 373-109.

[5] F. J. Mancebo, J. A. Nicolás and J. M. Vega, Chaotic oscillations in a nearly-inviscid axisymmetric capillary bridge at 2: 1 parametric resonance. Fhys. Flvids 10 (1998), 10881103.

[6] D. J. Mollot, J. 'Isamopoulos, 'I'. Y. Chen and A. Ashgriz, Nonlinear dynamics of capillary bridges: experiments. J. Fluid Mech 255 (1993), 411-435.

[7] A. V. Anilkumar, R. N. Grugel, X. F. Shen, C. P. Tee and T. C. Wang, Control of thermocapillary convection in a liquid bridge by vibration. J. Appl. Phys. 73 (1993), 1165-1170.

[8] J. $\Lambda$. Nicolés, D. Rivas and J. M. Vegen, The interaction of thermocapillary convection and low-frequency vibration in nearly-inviseid liquid bridges. Z. Angew. Math. Phys. 48 (1997), 389-423.

[9] J. A. Nicolás, L. Rivas and J. M. Vega, On the stearly streaming flow due to high frequency vibration in nearly-inviscid liquid bridges. J. Fluid Mech. 354 (1998), 1/7-17/.

[10] K. M. Case and W. C. Parkinson, Damping of surface waves in an incompressible liquid. J. Fluid Mech. 2 (1957), 172184.

[11] D. M. IIenderson and J. W. Miles, Single mode Faraday waves in small cylinders. J. Fintid Mech, $213(1990), 95-110$.

[12] D. M. Henderson and J. W. Miles, Surface-wave damping in a circular cylinder with a fixed contact line. I. Flusd Mech. 275 (1991), 285-299.

113| C. Martel, J. A. Nicolás and J. M. Vega, Surface-wave damping in a brimful cylindrical container. J. Fluid Mech. 360 (1998), 213 228. See also Corrigendum, 373 (1998), 379.

[14] J. W. Miles and D. M. IIenderson, $A$ note on interior vs. boundary-layer damping of surface waves in a circular cylinder. J. Firid Mech. 264 (1998), 310-323.

[15] J. A. Nicolás and J. Miles, A note on damping of capillary-gravity waves in a brimful circular cylinder. Submitted to J. Fivid Mech, 1900.

[16] J. A. Nicolás and J. M. Vega, A note on the effect of surface contamination in water wave damping. J. Fluid Mech., in press, 2000.

[17] $\Lambda$. Sanz, The inntuence of the outer bath in the dynarries of axisymmetric licquid bridgess. J. Fluid Mech. $156(1985), 101140$.

[18] A. Sanz and J. L. D'ez, Non-axisymmetric oscillations of liquid bridges. I. Fibid Mech. $205(1989), 503-521$.

[19] A. Borkar and J. Tsamopoulos, Boundary layer analysis of the dynamics of axisymmetric capillary bridges. Phys. Fhusds A $3(1991), 2866-2874$.

[20] M. Iliguera, J. A. Nicolás and J. M. Vega, Lincar oscillations of woakly dissipalive axisymmetric licuid bridges. Fhys. Fluids A 6 (1994), 438450.

[21] M. Higuera and J. A. Nicolás, Linear non-axisymmetric oscillations of nearly-inviscid liquid bridges. Phas. Fivids 9 (1997), 276-285.

[22] J. A. Nicolás, Hydrodynamic stability of high-viscosity cylindrical liquid bridges. Phys. Fhisids A 4 (1992), 1620-1626.

[23] J. Tsarropoulos, T. Y. Chen and $\Lambda$. Borkar, Viscous oscillations of capillary bridges. $J$. Fluid Mech. 235 (1992), 579609.

$[24 \mid$ D. D. Joseph and L. Sturges, 'The free surface of a liquid filling a trench heated from its side. I. Filuid Mech. 69 (1975), 565-589.

[25] D. D. Joseph, The convergence of biorthogonal series for biharmonic and Stokes flow edge problems, Part I. SIAM J. Appl. Math. 33 (1977), 337-347.

[26] D. D. Joseph and L. Sturges, The convergence of biorthogonal series for biharmonic and Stokes flow edge problems, Part II. SIAM J. Appl. Math. 34 (1978), 726.

[27] R. C. 'T. Smith, 'The bending of a semi-infinite strip. Austral. J. Sci. Res. 5 (1952), $227-237$. 
[28] J. Graharn-Eagle, $\Delta$ new method for calculating eigenvalues with applications to gravitycapillary waves with edge constrains. Math. I'roc. Camb. I'hit. Soe 94 (1083), 553-564.

[20] F. Ursell, Infinite systems of equations. 'T'he effect of truncation. Q. Jl. Appl. Math. 49 (1996), 217-223.

[30] H. B. Keller, Lectures on Numerical Methods in Bifurcation Problems. Springer-Verlag, Tata Institute of Fundamental Researeh, Borrbay, 1987.

[31] F. J. Garcik and $A$. Castellanos, One-dimensional models for slender axisymmetric viscous licuid bridges. Thas. Fluids 8 (1096), $2837-2846$.

[32] 1. S. Gradshteyn and 1. M. Ryzhik, Table of Integrols, Series and l'roducts. Academic Press, 1980.

[33| F. Traacson and H. B. Keller, Anahysis of Numerical Methods. John Wiley, 1966.

[34] II. Lamb, IIydrodynamics. Carrbridge Universily Press, 1932.

[35] II. Schlichting, Berechnung ebener periodischer Grenzschichtströmungen. Phys. Z. 33 (1932), 327-335.

[36] M. s. Longuet-Higgins, Mass transport in water waves. Thit. Trons. Hoy. Soc. A $\mathbf{2 4 5}$ (1953), $535-581$.

José $A$. Nicolás and José M. Vega

E.'I's.l. Aeronáuticos

Universidad l'olitécnica de Marlrid

Madrid, Spain

(e-mail: vega(ufmetsia.upm.es) 